\title{
Singular Perturbation Theory for Homoclinic Orbits in a Class of Near-Integrable Hamiltonian Systems*
}

\author{
Gregor Kovačič \\ Mathematical Sciences Department \\ Rensselaer Polytechnic Institute \\ Troy, NY 12180
}

\begin{abstract}
This paper describes a new type of orbits homoclinic to resonance bands in a class of near-integrable Hamiltonian systems. It presents a constructive method for establishing whether small conservative perturbations of a family of heteroclinic orbits that connect pairs of points on a circle of equilibria will yield transverse homoclinic connections between periodic orbits in the resonance band resulting from the perturbation. In any given example, this method may be used to prove the existence of such transverse homoclinic orbits, as well as to determine their precise shape, their asymptotic behavior, and their possible bifurcations. The method is a combination of the Melnikov method and geometric singular perturbation theory for ordinary differential equations.
\end{abstract}

\section{Introduction}

Homoclinic chaos is a very common phenomenon in two-degree-of-freedom Hamiltonian systems. For those systems that are close to being integrable, its presence can be established by the well known Melnikov method, first developed for this particular situtation in Holmes and Marsden [1982] and Robinson [1988], and then systematically extended to $n$-degree-offreedom systems in the book by Wiggins [1988]. The common feature of all these studies is that they deal with families of unstable periodic orbits that are connected to themselves by transverse homoclinic orbits. Via the Smale-Birkhoff homoclinic theorem (Guckenheimer and Holmes [1983]), this situation leads to chaotic dynamics of phase points in the vicinity

*Appeared in Journal of Dynamics and Differential Equations 5 (1993), 559-597. 
of the homoclinic orbits. All the above studies explicitly exclude the case of resonance, in which the frequency of one of the periodic orbits vanishes.

This current paper takes the complementary approach in that it focuses exclusively on the resonant case. The principal result of this paper is presented in the Main Theorem in section 7. This theorem describes a constructive method that can be used to verify whether the stable and unstable manifolds of two periodic orbits in a resonance band intersect transversely inside the corresponding level surface of the Hamiltonian. This method is completely general, and more importantly, very simple to use. As opposed to the usual Melnikov theory, which gives the Melnikov function as an integral, an idea of Feng [1990] makes it possible to write down the Melnikov function at the resonance in closed form, and even to make certain that it always has zeros. Thus, in a specific application, one needs only to use the general formulas presented in this paper's Main Theorem, in order to ascertain whether the problem at hand possesses orbits homoclinic to a resonance band.

The difficulty in deriving the Main Theorem arises because a resonance band is a structure born under perturbation out of a closed curve of equilibria. Pairs of equilibria on this curve are connected to each other by heteroclinic orbits. This paper determines the deformations of these heteroclinic orbits under perturbation that result in heteroclinic connections between pairs of periodic orbits in the resonance band. The description we give of these deformations requires an approach from singular perturbation theory. In particular, the analysis is performed in three steps. The first step, described in section 4, uses the Melnikov method (Poincaré [1899], Melnikov [1963], Arnold [1964], Chow, Hale, and MalletParet [1980], Holmes and Marsden [1982], Guckenheimer and Holmes [1983], Robinson [1988], Wiggins [1988]) to establish the survival under perturbation of some of the heteroclinic orbits that connect pairs of equilibria on the unperturbed closed curve to each other. The Melnikov method is a regular perturbation method, and only establishes the survival of heteroclinic orbits, not their precise shape or details of their asymptotic behavior. To find these, we must perform the second step, described in section 5, which, by use of a proper rescaling, analyzes the singular problem of how a closed curve of equilibria deforms under perturbation into a resonance band, containing mainly nested families of periodic orbits (see Greenspan and Holmes [1984], Guckenheimer and Holmes [1983], Arnold [1988], Wiggins [1990]). The third and final step, developed in sections 6 and 7, then uses geometric singular perturbation theory due to Fenichel [1979] (see also Sakamoto [1990], and Jones and Kopell [1992]) to couple the dynamics of the homoclinic or heteroclinic orbits with the dynamics in the resonance band. 
Orbits homoclinic to resonance bands arise in many physical models, particularly those models that are obtained by transforming coordinates into a rotating reference frame and averaging over a fast phase. Examples of such models are Bishop, Flesch, Forest, McLaughlin, and Overman [1990] in the theory of Josephson's junctions; David, Holm, and Tratnik [1990] in nonlinear fiber optics; Holm and Kovačič [1992] in laser-matter interaction; Holmes [1986], Gu and Sethna [1987], and Feng and Sethna [1989], [1990] in the theory of water waves; and Yang and Sethna [1991] in the theory of vibrating plates. All the resonant periodic motions of such physical models, that is, periodic motions whose frequencies coincide with the frequency of the rotating frame, become circles of equilibria. These circles are usually embedded in a one-parameter family of periodic orbits. If this family is unstable and connected to itself by a homoclinic manifold, which is a common occurrence, the theory presented in this paper applies to it.

Resonance bands and orbits homoclinic to them are much more likely to survive the addition of dissipation to the problem than are families of periodic orbits and orbits homoclinic to these periodic orbits. In particular, it is likely that in some dissipative systems orbits homoclinic to resonance bands are the only source of chaotic dynamics in the phase space. (A special case of the theory of orbits homoclinic to resonance bands in a dissipative system was first discussed in Kovačič and Wiggins [1992], a general theory is presented in Kovačič [1992c]. Examples, special cases, and parallel developments of the theory are given in Feng and Wiggins [1992], Kovačič [1992b], and McLaughlin et. al. [1993].) While the dynamics of orbits homoclinic to resonace bands in dissipative cases are quite different from those in the nondissipative case, the methods of analysis in the two cases have many similarities.

The paper is organized as follows: In section 2 we present the class of systems under investigation, describe its related unperturbed problem, and make the basic assumptions that identify a problem as that of orbits homoclinic to a resonance band. In section 3 we describe the background material we need on persistence of normally hyperbolic invariant manifolds. In section 4 we describe the Melnikov method used in our problem and show how the corresponding Melnikov function can be computed in closed form. In section 5 we introduce the rescaled system that describes the dynamics in the resonance bands. In section 6 we describe the tools from geometric singular perturbation theory we need and apply them to the computation of the local stable and unstable manifolds of periodic orbits and equilibria contained in resonance bands. In section 7 we use the results from sections 4, 5 and 6 to derive the Main Theorem. In section 8 we illustrate the Main Theorem on a simple example, and in section 9 we show how to extend the present work to higher dimensions in 
the context of another example.

\section{The Setup}

We consider two-degree-of-freedom Hamiltonian systems with perturbations that break an $S^{1}$ phase symmetry. These systems appear in the following form:

$$
\begin{gathered}
\dot{x}=J D_{x} H_{0}(x, I)+\varepsilon J D_{x} H_{1}(x, I, \theta), \\
\dot{I}=-\varepsilon D_{\theta} H_{1}(x, I, \theta), \\
\dot{\theta}=D_{I} H_{0}(x, I)+\varepsilon D_{I} H_{1}(x, I, \theta),
\end{gathered}
$$

with $x=\left(x_{1}, x_{2}\right) \in \mathbf{R}^{2}, I \in \mathbf{R}, \theta \in S^{1}$ and $J=\left(\begin{array}{cc}0 & -1 \\ 1 & 0\end{array}\right)$. Here $D_{x}=\left(D_{x_{1}}, D_{x_{2}}\right)$ represents partial derivatives with respect to $x ; D_{I}$ represents the partial derivative with respect to $I$; and $D_{\theta}$ represents the partial derivative with respect to $\theta$. Moreover, $0<\varepsilon \ll 1$ is a small parameter. This system is derived from the Hamiltonian

$$
H(x, I, \theta, \varepsilon)=H_{0}(x, I)+\varepsilon H_{1}(x, I, \theta) .
$$

We call the system (2.1) with $\varepsilon=0$ and the Hamiltonian (2.2) with $\varepsilon=0$ the unpertrubed system and the unperturbed Hamiltonian, respectively. The unperturbed system

$$
\begin{gathered}
\dot{x}=J D_{x} H_{0}(x, I), \\
\dot{I}=0, \\
\dot{\theta}=D_{I} H_{0}(x, I)
\end{gathered}
$$

can be derived from the unperturbed Hamiltonian $H_{0}(x, I)$. Because of the phase symmetry in $\theta$, system (2.3) is completely integrable; that is, its solutions can be obtained by quadratures. Namely, equation (2.3a) is a planar Hamiltonian system for the variable $x=\left(x_{1}, x_{2}\right)$, since $I$ is a constant. The variable $\theta$ can be obtained by a quadrature from $(2.3 \mathrm{c})$ once the solution for $x$ is known. In the language of classical mechanics, $I$ is an action and $\theta$ is its conjugate angle.

For the rest of this paper we assume

Assumption 1 Equation (2.3a) has a hyperbolic equilibrium at $x=X(I)$ for all $I$ with $I_{1}<I<I_{2}$ for some $I_{1}$ and $I_{2}$. Furthermore, the equilibrium $X(I)$ is connected to itself by a homoclinic orbit $x^{h}(t, I)$, that is, $\lim _{t \rightarrow \pm \infty} x^{h}(t, I)=X(I)$. 
Since the equilibrium $X(I)$ is hyperbolic, that is, the eigenvalues of the stability matrix $J D_{x}^{2} H_{0}(X(I), I)$ are both nonzero, all the equilibria $X(I)$ with $I_{1}<I<I_{2}$ form a smooth curve of equilibria in the $x-I$ space. Moreover, in the full $x-I-\theta$ phase space, each equilibrium $X(I)$ corresponds to a periodic orbit $O^{I}$ parametrized by the solution

$$
x=X(I), \quad I=I, \quad \theta=D_{I} H_{0}(X(I), I) t+\theta_{0} .
$$

Another assumption that we make is

\section{Assumption 2 The twist condition}

$$
\frac{d}{d I}\left[D_{I} H_{0}(X(I), I)\right]=D_{x} D_{I} H_{0}(X(I), I) \frac{d X(I)}{d I}+D_{I}^{2} H_{0}(X(I), I) \neq 0 .
$$

is fulfilled on all the periodic orbits, that is, for all $I_{1}<I<I_{2}$.

This condition implies that the frequency of the periodic orbits $O^{I}$ changes from one orbit to another.

Each orbit $O^{I}$ is hyperbolic inside the corresponding level surface of the Hamiltonian $H_{0}$, and possesses two-dimensional stable and unstable manifolds $W^{s}\left(O^{I}\right)$ and $W^{u}\left(O^{I}\right)$. Two branches of the manifolds $W^{s}\left(O^{I}\right)$ and $W^{u}\left(O^{I}\right)$ coincide to form a two-dimensional homoclinic manifold $W\left(O^{I}\right)$.

We also assume

Assumption 3 For some $I_{0}$ with $I_{1}<I_{0}<I_{2}$ the frequency of the corresponding periodic orbit $O^{I_{0}}$ vanishes, that is,

$$
D_{I} H_{0}\left(X\left(I_{0}\right), I_{0}\right)=0
$$

The twist condition (2.5) implies that this zero is simple, that is, $\frac{d}{d I}\left[D_{I} H_{0}\left(X\left(I_{0}\right), I_{0}\right)\right] \neq 0$. In this case, we refer to the value $I=I_{0}$ as a resonant $I$ value. Thus, at the resonant value $I_{0}$, the corresponding $O^{I_{0}}$ is a circle of equilibria.

Our last assumption is

Assumption 4 All the zeros (in $\theta$ ) of the derivative $D_{\theta} H_{1}\left(X\left(I_{0}\right), I_{0}, \theta\right)$ are simple. 


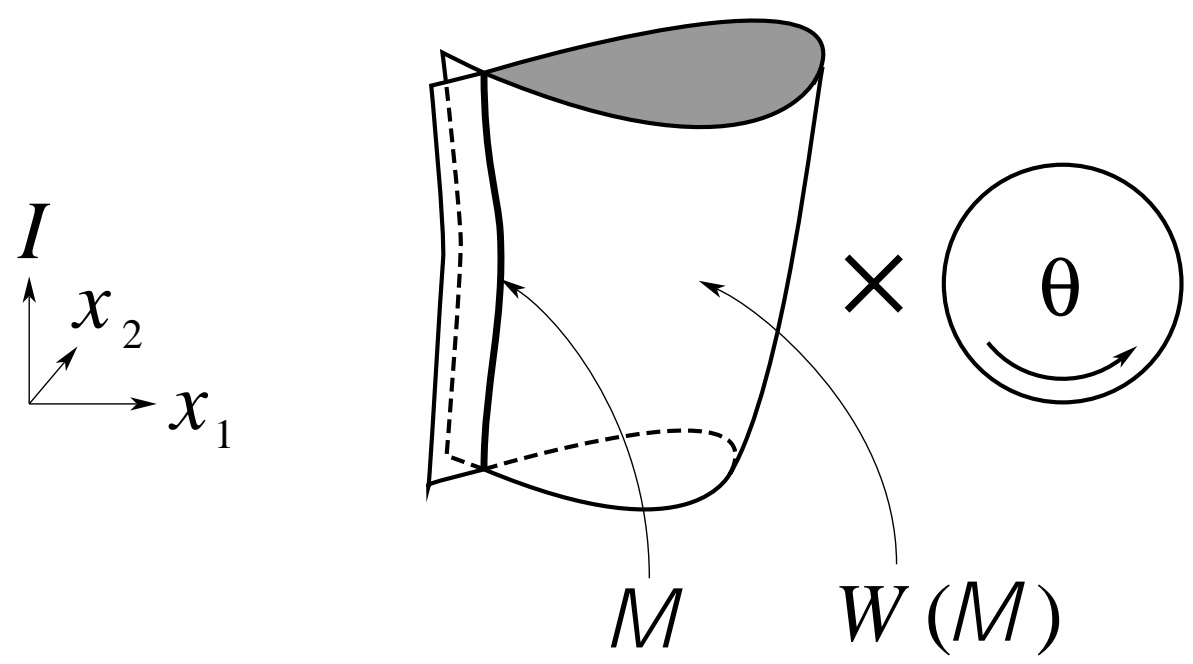

Figure 1: Geometry of the invariant annulus $\mathcal{M}$ and its homoclinic manifold $W(\mathcal{M})$.

The significance of this assumption will be made clear in section 5 .

The union of the orbits $O^{I}$ with $I_{1}<I<I_{2}$ is a two-dimensional annular invariant surface, $\mathcal{M}$, which is normally hyperbolic (for a precise definition and proof see Wiggins [1988]). Intuitively, this means that orbits on its stable and unstable manifolds, $W^{s}(\mathcal{M})$ and $W^{u}(\mathcal{M})$, approach $\mathcal{M}$ exponentially in forward and backward time, respectively, and that this approach is fast compared to the (linear) motion on $\mathcal{M}$ itself.

Two branches of the stable and unstable manifolds, $W^{s}(\mathcal{M})$ and $W^{u}(\mathcal{M})$ of the invariant annulus $\mathcal{M}$ coincide to form the three-dimensional homoclinic manifold $W(\mathcal{M})$, shown in figure 1 , which is the union over $I$ of the homoclinic manifolds $W\left(O^{I}\right)$. A parametrization of the homoclinic manifold $W(\mathcal{M})$ is provided by the solutions on it. In particular, this parametrization of $W(\mathcal{M})$ is given by

$$
(x, I, \theta)=\left(x^{h}(t, I), I, \theta^{h}\left(t, I, \theta_{0}\right)\right),
$$

where $x^{h}(t, I)$ is a particular trajectory on the orbit homoclinic to the point $x=X(I)$ in the equation $(2.3 \mathrm{a})$, and

$$
\theta^{h}\left(t, I, \theta_{0}\right)=\int_{0}^{t} D_{I} H_{0}\left(x^{h}(s, I), I\right) d s+\theta_{0} .
$$

(We here tacitly assume that the choice of the trajectory $x^{h}(t, I)$ varies smoothly with $I$.) The parameters of the manifold $W(\mathcal{M})$ in $(2.7)$ are $t, I$ and $\theta_{0}$. Alternatively, the homoclinic manifold $\mathcal{M}$ is described implicitly by the equation

$$
H_{0}(x, I)-H_{0}(X(I), I)=0
$$



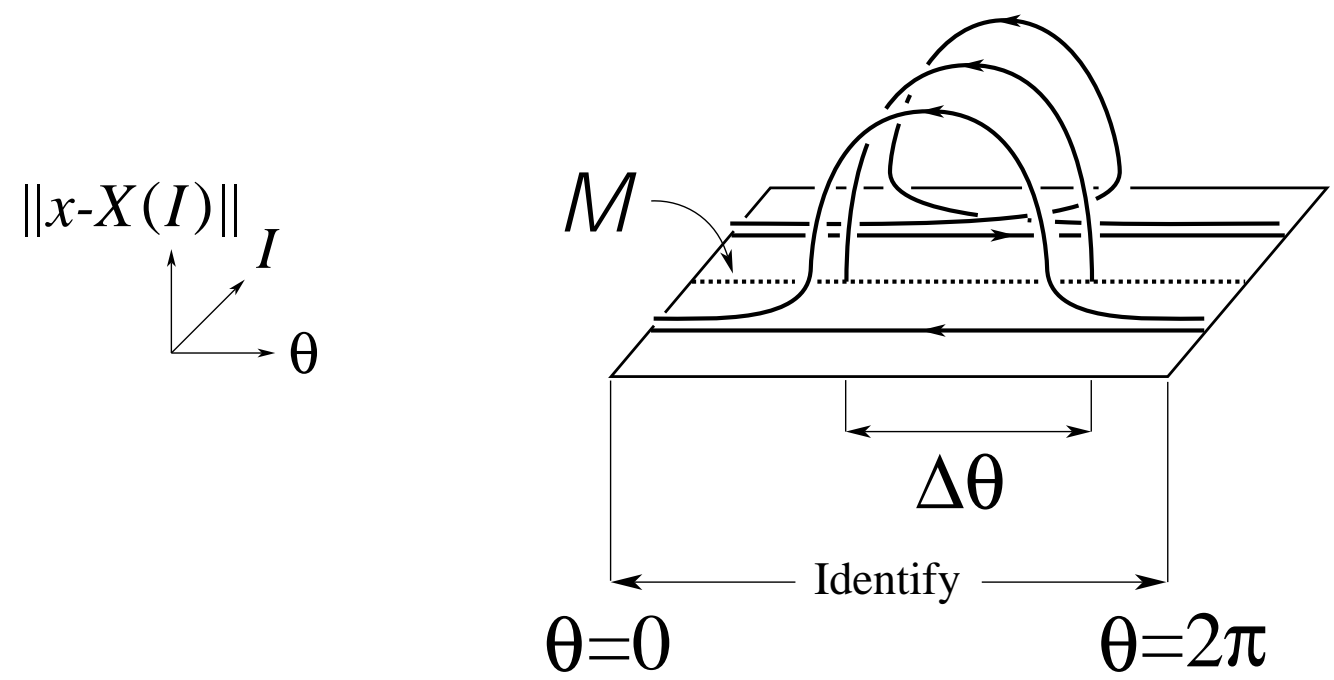

Figure 2: Geometry of manifolds homoclinic to periodic orbits and the circle of equilibria at $I=I_{0}$. Only one orbit is shown from each such manifold. All the other orbits on the same homoclinic manifold are obtained by translating those shown in the picture along the $\theta$-axis. Orbits on the manifold homoclinic to the circle of equilibria at $I=I_{0}$ are heteroclinic orbits connecting pairs of points on that circle that are $\Delta \theta$ apart.

which holds because the value of the Hamiltonian function $H_{0}$ on any homoclinic orbit must, by continuity, be equal to the value of $H_{0}$ on the object to which the orbit is homoclinic.

Individual homoclinic manifolds $W\left(O^{I}\right)$ are either parametrized by the expression $(2.7)$ with the value of $I$ fixed, or described implicitly by the equations

$$
H_{0}(x, I)-H_{0}(X(I), I)=0, \quad I=\text { constant. }
$$

When $O^{I}$ is a periodic orbit, the manifold $W\left(O^{I}\right)$ is a "pinched" two-torus of orbits homoclinic to $O^{I}$. (That is the cartesian product of an $\alpha$-shaped curve and a circle.)

For $I=I_{0}$, orbits on the homoclinic manifold $W\left(O^{I_{0}}\right)$ are generally heteroclinic orbits connecting pairs of points on the circle of equilibria $O^{I_{0}}$, see figure 2 . The shift in phase between the two end points of any such heteroclinic orbit is given by the expression

$$
\Delta \theta=\int_{-\infty}^{\infty} D_{I} H_{0}\left(x^{h}\left(s, I_{0}\right), I_{0}\right) d s,
$$

and is, since the unperturbed Hamiltonian $H_{0}$ does not depend on the angle $\theta$, independent of the particular choice of the heteroclinic orbit. The difference $\Delta \theta$ is always finite since $x^{h}\left(s, I_{0}\right) \rightarrow X\left(I_{0}\right)$ exponentially as $s \rightarrow \pm \infty$ and $D_{I} H_{0}\left(X\left(I_{0}\right), I_{0}\right)=0$. When the phase difference $\Delta \theta$ is an integer multiple of $2 \pi$, all the orbits on the manifold $W\left(O^{I_{0}}\right)$ are homoclinic. Otherwise, they are heteroclinic. 


\section{Persistence of Invariant Manifolds}

Much of the structure described in the previous section is sufficiently robust to persist under perturbations. As is shown in Wiggins [1988], the invariant manifold theory due to Fenichel [1971], [1974], [1977] can be adapted to show that the invariant annulus $\mathcal{M}$ persists together with its stable and unstable manifolds.

In particular, we have

Proposition 3.1 An invariant annulus $\mathcal{M}_{\varepsilon}$ persists $\mathcal{O}(\varepsilon)$ close to $\mathcal{M}$. This annulus can be written as a smooth graph, $x=X_{\varepsilon}(I, \theta)=X(I)+\varepsilon X_{1}(I, \theta)+\mathcal{O}\left(\varepsilon^{2}\right)$, over the unperturbed annulus $\mathcal{M}$. Away from $I=I_{0}$, the annulus $\mathcal{M}_{\varepsilon}$ is foliated by periodic orbits, which collapse smoothly onto the unperturbed periodic orbits on $\mathcal{M}$ as $\varepsilon \rightarrow 0$. Moreover, two of those periodic orbits form the boundary of $\mathcal{M}_{\varepsilon}$.

PROOF: As a first step, the existence of the annulus $\mathcal{M}_{\varepsilon}$ is proven using the Fenichel theory as described in Wiggins [1988]. This theory also implies smooth dependence of the annulus $\mathcal{M}_{\varepsilon}$ on $\varepsilon$, including the $\mathcal{O}(\varepsilon)$ estimate of the distance between the annuli $\mathcal{M}_{\varepsilon}$ and $\mathcal{M}$. However, even though this step guarantees that $\mathcal{M}_{\varepsilon}$ is spanned by segments of orbits, this annulus need not be invariant, since it may "leak out" phase points along its boundary at $I=I_{1}$ and $I=I_{2}$. (It must be locally invariant; see Wiggins [1988].) We now show how to guarantee that $\mathcal{M}_{\varepsilon}$ is invariant and, in the same breath, prove the rest of the proposition.

For nonresonant $I$, that is $I \neq I_{0}$, the annulus $\mathcal{M}$ and the unperturbed surfaces of constant Hamiltonian $H_{0}$ intersect transversely along the periodic orbits $O^{I}$. The perturbed annulus $\mathcal{M}_{\varepsilon}$ is $\mathcal{O}(\varepsilon)$ close the unperturbed annulus $\mathcal{M}$, and the level surfaces of the perturbed Hamiltonian $H$ are $\mathcal{O}(\varepsilon)$ close to the level surfaces of the unperturbed Hamiltonian $H_{0}$. Therefore, the perturbed annulus $\mathcal{M}_{\epsilon}$ intersects the level surfaces of the perturbed Hamiltonian $H$ transversely along perturbed periodic orbits $O_{\varepsilon}^{I}$. (Here we denote by $O_{\varepsilon}^{I}$ the perturbed periodic orbit having the same frequency as the unperturbed orbit $O^{I}$, which is possible because we assume that the twist condition $\frac{d}{d I}\left[D_{I} H_{0}(X(I), I)\right] \neq 0$ is fulfilled. We could equally well denote by $O_{\varepsilon}^{I}$ the perturbed periodic orbit on which the perturbed Hamiltonian $H$ would attain the same value as the unperturbed Hamiltonian $H_{0}$ does on $O^{I}$. In both cases, the perturbed orbit $O_{\varepsilon}^{I}$ collapses smoothly onto the unperturbed orbit $O^{I}$ as $\varepsilon \rightarrow 0$. The choice of frequency as the characterization of the orbit $O_{\varepsilon}^{I}$ is more convenient, since it implies the sharp version of proposition 3.4 stated below.) We can now choose two perturbed periodic orbits on the perturbed surface $\mathcal{M}_{\varepsilon}$, one $\mathcal{O}(\varepsilon)$ close to $I=I_{1}$, and the 
other $\mathcal{O}(\varepsilon)$ close to $I=I_{2}$, as the boundaries of $\mathcal{M}_{\varepsilon}$. The perturbed annulus $\mathcal{M}_{\varepsilon}$ thus becomes invariant.

Fenichel's persistence theory implies that the perturbed annulus $\mathcal{M}_{\varepsilon}$ possesses (threedimensional) local stable and unstable manifolds, $W_{l o c}^{s}\left(\mathcal{M}_{\varepsilon}\right)$ and $W_{l o c}^{u}\left(\mathcal{M}_{\varepsilon}\right)$. For any small enough but finite $\delta$, these manifolds are defined as the sets of trajectories that start in the $\delta$ neighborhood of the unperturbed annulus $\mathcal{M}$, never leave this neighborhood and approach the perturbed annulus $\mathcal{M}_{\varepsilon}$ in forward and backward time, respectively. The precise statement concerning the manifolds $W_{\text {loc }}^{s}\left(\mathcal{M}_{\varepsilon}\right)$ and $W_{l o c}^{u}\left(\mathcal{M}_{\varepsilon}\right)$ is contained in

Proposition 3.2 The perturbed annulus $\mathcal{M}_{\varepsilon}$ possesses three-dimensional local stable and unstable manifolds, $W_{\text {loc }}^{s}\left(\mathcal{M}_{\varepsilon}\right)$ and $W_{\text {loc }}^{u}\left(\mathcal{M}_{\varepsilon}\right)$. Orbits in $W_{\text {loc }}^{s}\left(\mathcal{M}_{\varepsilon}\right)$ approach $\mathcal{M}_{\varepsilon}$ exponentially fast in forward time, and orbits in $W_{\text {loc }}^{u}\left(\mathcal{M}_{\varepsilon}\right)$ approach $\mathcal{M}_{\varepsilon}$ exponentially fast in backward time. The local stable and unstable manifolds, $W_{l o c}^{s}\left(\mathcal{M}_{\varepsilon}\right)$ and $W_{\text {loc }}^{u}\left(\mathcal{M}_{\varepsilon}\right)$, together with their tangent spaces, vary smoothly with $\varepsilon$ (and any other parameters in the problem), and in particular, collapse smoothly onto their unperturbed counterparts, the local stable and unstable manifolds, $W_{\text {loc }}^{s}(\mathcal{M})$ and $W_{\text {loc }}^{u}(\mathcal{M})$, and their tangent spaces, as $\varepsilon \rightarrow 0$.

The global stable and unstable manifolds, $W^{s}\left(\mathcal{M}_{\varepsilon}\right)$ and $W^{u}\left(\mathcal{M}_{\varepsilon}\right)$ of the perturbed annulus $\mathcal{M}_{\varepsilon}$ are now obtained by evolving initial conditions contained in the local manifolds, $W_{l o c}^{s}\left(\mathcal{M}_{\varepsilon}\right)$ and $W_{l o c}^{u}\left(\mathcal{M}_{\varepsilon}\right)$, in forward and backward time, respectively.

Using a transversality argument similar to that in the proof of proposition 3.1 we infer

Proposition 3.3 Away from $I=I_{0}$, the three-dimensional local stable and unstable manifolds $W_{\text {loc }}^{s}\left(\mathcal{M}_{\varepsilon}\right)$ and $W_{\text {loc }}^{u}\left(\mathcal{M}_{\varepsilon}\right)$ of the perturbed annulus $\mathcal{M}_{\varepsilon}$ are foliated by surfaces of constant Hamiltonian $H$ into the local stable and unstable manifolds $W_{l o c}^{s}\left(O_{\varepsilon}^{I}\right)$ and $W_{l o c}^{u}\left(O_{\varepsilon}^{I}\right)$ of the perturbed periodic orbits $O_{\varepsilon}^{I}$.

Consequently, the three-dimensional global stable and unstable manifolds $W^{s}\left(\mathcal{M}_{\varepsilon}\right)$ and $W^{u}\left(\mathcal{M}_{\varepsilon}\right)$ of the perturbed annulus $\mathcal{M}_{\varepsilon}$ are foliated by surfaces of constant Hamiltonian $H$ into the global stable and unstable manifolds $W^{s}\left(O_{\varepsilon}^{I}\right)$ and $W^{u}\left(O_{\varepsilon}^{I}\right)$ of the perturbed periodic orbits $O_{\varepsilon}^{I}$.

Finally, simple Gronwall-type estimates imply

Proposition 3.4 Away from $I=I_{0}$, any trajectory on the perturbed stable manifold $W^{s}\left(O_{\varepsilon}^{I}\right)$ stays $\mathcal{O}(\varepsilon)$ close to a trajectory on the unperturbed stable manifold $W^{s}\left(O^{I}\right)$ on the semi- 
infinite time interval $T<t<\infty$ for any real $T$, and any trajectory on the perturbed unstable manifold $W^{u}\left(O_{\varepsilon}^{I}\right)$ stays $\mathcal{O}(\varepsilon)$ close to a trajectory on the unperturbed unstable manifold $W^{u}\left(O^{I}\right)$ on the semi-infinite time interval $-\infty<t<T$ for any real $T$. More generally, any trajectory on the perturbed stable manifold $W^{s}\left(\mathcal{M}_{\varepsilon}\right)$ stays $\mathcal{O}(\varepsilon)$ close to a trajectory on the unperturbed stable manifold $W^{s}(\mathcal{M})$, and any trajectory on the perturbed unstable manifold $W^{u}\left(\mathcal{M}_{\varepsilon}\right)$ stays $\mathcal{O}(\varepsilon)$ close to a trajectory on the unperturbed unstable manifold $W^{u}(\mathcal{M})$, both on the time interval $-T<t<T$ for any positive $T$.

\section{Homoclinic Intersections}

We now begin investigating what becomes of the structure homoclinic to the annulus $\mathcal{M}$ under perturbation. Genericity considerations reveal that it is not reasonable to expect two branches of the stable and unstable manifolds, $W^{s}\left(\mathcal{M}_{\varepsilon}\right)$ and $W^{u}\left(\mathcal{M}_{\varepsilon}\right)$, of the perturbed surface $\mathcal{M}_{\varepsilon}$ to coincide and form a three-dimensional homoclinic manifold. Namely, both $W^{s}\left(\mathcal{M}_{\varepsilon}\right)$ and $W^{u}\left(\mathcal{M}_{\varepsilon}\right)$ are three-dimensional, and in the four-dimensional $x-I-\theta$ ambient space, their generic transverse intersections are expected to be $3+3-4=2$ dimensional. Since the unperturbed intersection $W(\mathcal{M})$ of the manifolds $W^{s}(\mathcal{M})$ and $W^{u}(\mathcal{M})$ is not transverse, we thus expect the perturbed manifolds $W^{s}\left(\mathcal{M}_{\varepsilon}\right)$ and $W^{u}\left(\mathcal{M}_{\varepsilon}\right)$ to either intersect transversely along two-dimensional (homoclinic) submanifolds, or to not intersect at all, except of course along the annulus $\mathcal{M}_{\varepsilon}$ itself. We check for the existence of transverse homoclinic intersections using the Melnikov method. This method is standard, and we therefore only briefly review those of its features that will be important for the current paper. All the relevant details are described in Wiggins [1988].

We check for the intersections of the manifolds $W^{s}\left(\mathcal{M}_{\varepsilon}\right)$ and $W^{u}\left(\mathcal{M}_{\varepsilon}\right)$ by calculating the distance between appropriately chosen pairs of points $a_{\varepsilon}^{s}$ and $a_{\varepsilon}^{u}$, with $a_{\varepsilon}^{s}$ lying on $W^{s}\left(\mathcal{M}_{\varepsilon}\right)$, and $a_{\varepsilon}^{u}$ lying on $W^{u}\left(\mathcal{M}_{\varepsilon}\right)$. The manifolds $W^{s}\left(\mathcal{M}_{\varepsilon}\right)$ and $W^{u}\left(\mathcal{M}_{\varepsilon}\right)$ intersect where this distance vanishes. The appropriate pairs of points $a_{\varepsilon}^{s}$ and $a_{\varepsilon}^{u}$ are found in the following way. We first use the unperturbed homoclinic manifold $W(\mathcal{M})$ as the framework for parametrizing the manifolds $W^{s}\left(\mathcal{M}_{\varepsilon}\right)$ and $W^{u}\left(\mathcal{M}_{\varepsilon}\right)$ as follows. At every point $a$ on the homoclinic manifold $W(\mathcal{M})$ we consider the normal $\mathbf{n}(a)$ to $W(\mathcal{M})$. The normal $\mathbf{n}(a)$ can be easily calculated from formula (2.9). If $a=(x, I, \theta)$, then

$$
\mathbf{n}(a)=\left(D_{x} H_{0}(x, I), D_{I} H_{0}(x, I)-D_{I} H_{0}(X(I), I), 0\right) .
$$

The normal $\mathbf{n}(a)$ pierces the manifold $W(\mathcal{M})$ transversely in precisely the point $a$. Therefore, $\mathbf{n}(a)$ also pierces the perturbed manifolds $W^{s}\left(\mathcal{M}_{\varepsilon}\right)$ and $W^{u}\left(\mathcal{M}_{\varepsilon}\right)$ transversely in discrete 
points, $\mathcal{O}(\varepsilon)$ away from the point $a$. Now, since the manifolds $W^{s}\left(\mathcal{M}_{\varepsilon}\right)$ and $W^{u}\left(\mathcal{M}_{\varepsilon}\right)$ may fold back onto themselves, they may intersect the normal $\mathbf{n}(a)$ in more than one point each. However, the unique choice of two points, $a_{\varepsilon}^{s} \in W^{s}\left(\mathcal{M}_{\varepsilon}\right)$ and $a_{\varepsilon}^{u} \in W^{u}\left(\mathcal{M}_{\varepsilon}\right)$, that is consistent with the perturbation theory is the following. The point $a_{\varepsilon}^{s}$ is the unique intersection point of the normal $\mathbf{n}(a)$ and the stable manifold $W^{s}\left(\mathcal{M}_{\varepsilon}\right)$ inside an $\mathcal{O}(\varepsilon)$ neighborhood of $a$, whose trajectory takes the least amount of time to reach the local stable manifold $W_{l o c}^{s}\left(\mathcal{M}_{\varepsilon}\right)$ of the annulus $\mathcal{M}_{\varepsilon}$. We choose the point $a_{\varepsilon}^{u}$ in an analogous manner.

We now give an approximate expression for the distance between the two points $a_{\varepsilon}^{s}$ and $a_{\varepsilon}^{u}$, namely, the signed length of the vector $a_{\varepsilon}^{u}-a_{\varepsilon}^{s}$. If this distance vanishes, the manifolds $W^{s}\left(\mathcal{M}_{\varepsilon}\right)$ and $W^{u}\left(\mathcal{M}_{\varepsilon}\right)$ intersect at the point $a_{\varepsilon}^{s}=a_{\varepsilon}^{u}$. Since the vector $a_{\varepsilon}^{u}-a_{\varepsilon}^{s}$ lies along the normal $\mathbf{n}(a)$, its signed length is equal to

$$
d(a, \varepsilon)=\frac{\left\langle a_{\varepsilon}^{u}-a_{\varepsilon}^{s}, \mathbf{n}(a)\right\rangle}{\|\mathbf{n}(a)\|},
$$

where $\langle\cdot, \cdot\rangle$ denotes the usual Euclidean scalar product and $\|\cdot\|$ denotes the corresponding Euclidean norm. The quantity $d(a, \varepsilon)$ is thus equal to the signed distance between the points $a_{\varepsilon}^{s}$ and $a_{\varepsilon}^{u}$. The distance $d(a, \varepsilon)$ can be Taylor expanded (see Holmes and Marsden [1982], Lerman and Umanski [1984], Robinson [1988] or Wiggins [1988]) to become

$$
d(a, \varepsilon)=\varepsilon \frac{M\left(I, \theta_{0}\right)}{\|\mathbf{n}(a)\|}+\mathcal{O}\left(\varepsilon^{2}\right) .
$$

The expression

$$
M\left(I, \theta_{0}\right)=\int_{-\infty}^{\infty}\langle\mathbf{n}(a(t)), \mathbf{g}(a(t))\rangle d t
$$

is called the Melnikov function. Here,

$$
\mathbf{g}(x, I, \theta)=\left(J D_{x} H_{1}(x, I, \theta),-D_{\theta} H_{1}(x, I, \theta), D_{I} H_{1}(x, I, \theta)\right),
$$

and $a(t)$ is the unperturbed homoclinic orbit passing through the point $a$. The timeparametrization of this orbit $a(t)$ is given by formula (2.5). An application of the implicit function theorem to the quantity $\frac{d(a, \varepsilon)}{\varepsilon}$ shows that $d(a, \varepsilon)$ must vanish $\mathcal{O}(\varepsilon)$ close to a point $a$ where $M\left(I, \theta_{0}\right)$ vanishes, provided that at least one of its derivatives is nonzero. Moreover, the intersection of the manifolds $W^{s}\left(\mathcal{M}_{\varepsilon}\right)$ and $W^{u}\left(\mathcal{M}_{\varepsilon}\right)$ is then transverse. This is because, as formula (4.1) shows, the distance between $W^{s}\left(\mathcal{M}_{\varepsilon}\right)$ and $W^{u}\left(\mathcal{M}_{\varepsilon}\right)$ at the intersection point passes through zero with nonzero speed when we vary either $I$ (if the nonzero derivative is $D_{I} M\left(I, \theta_{0}\right)$ ) or $\theta_{0}$ (if the nonzero derivative is $D_{\theta_{0}} M\left(I, \theta_{0}\right)$ ).

We note that the Melnikov function $M\left(I, \theta_{0}\right)$ does not depend on the variable $t$. This is because the Melnikov function is constant along the unperturbed homoclinic or heteroclinic 
orbits. In particular, if $M\left(I, \theta_{0}\right)$ is zero at some point $a$, it must be zero along the whole orbit $a(t)$. This reflects the fact that the manifolds $W^{s}\left(\mathcal{M}_{\varepsilon}\right)$ and $W^{u}\left(\mathcal{M}_{\varepsilon}\right)$ are invariant. Therefore, if $W^{s}\left(\mathcal{M}_{\varepsilon}\right)$ and $W^{u}\left(\mathcal{M}_{\varepsilon}\right)$ intersect at a point, they must intersect along the whole orbit passing through that point, and $a(t)$ is a first approximation to this orbit, except possibly inside the local stable and unstable manifolds, $W_{l o c}^{s}\left(\mathcal{M}_{\varepsilon}\right)$ and $W_{l o c}^{u}\left(\mathcal{M}_{\varepsilon}\right)$. In fact, by proposition 3.4, this approximation is uniformly valid for orbits homoclinic to periodic orbits away from the resonance.

Recalling that the unperturbed homoclinic manifold is parametrized by three parameters, $I, \theta_{0}$ and $t$, we may expect that, because of the equation $M\left(I, \theta_{0}\right)=0$, which presents one constraint, intersections of the manifolds $W^{s}\left(\mathcal{M}_{\varepsilon}\right)$ and $W^{u}\left(\mathcal{M}_{\varepsilon}\right)$ are two-dimensional surfaces, parametrized by either $I$ and $t$ or $\theta_{0}$ and $t$, depending on whether $D_{I} M\left(I, \theta_{0}\right)$ or $D_{\theta_{0}} M\left(I, \theta_{0}\right)$ is nonzero. Thus, every intersection surface is a one-parameter family of orbits homoclinic to the perturbed annulus $\mathcal{M}_{\varepsilon}$. In particular, if we assume that $M\left(I, \bar{\theta}_{0}(I)\right)=0$ and that $D_{\theta_{0}} M\left(I, \bar{\theta}_{0}(I)\right)$ is nonzero, for all $I$ with $I_{1} \leq I \leq I_{2}$ and some function $\bar{\theta}_{0}(I)$, our perturbed system possesses a two-dimensional intersection surface $\Sigma_{\varepsilon}\left(\bar{\theta}_{0}\right)$ of the manifolds $W^{s}\left(\mathcal{M}_{\varepsilon}\right)$ and $W^{u}\left(\mathcal{M}_{\varepsilon}\right)$. This surface is parametrized by the parameters $I$ and $t$, while $\theta_{0}=\bar{\theta}_{0}(I)$.

At the resonance $I=I_{0}$, the Melnikov function can be computed explicitly (Feng [1990]). Before stating this result, we must first define the angle differences

$$
\Delta \theta_{+}=\int_{0}^{\infty} D_{I} H_{0}\left(x^{h}\left(s, I_{0}\right), I_{0}\right) d s, \quad \Delta \theta_{-}=\int_{-\infty}^{0} D_{I} H_{0}\left(x^{h}\left(s, I_{0}\right), I_{0}\right) d s .
$$

The integrals in these differences converge for the same reason as the integral (2.10) for $\Delta \theta$ does. In fact,

$$
\Delta \theta=\Delta \theta_{+}+\Delta \theta_{-}
$$

Now we can state

Proposition 4.1 The Melnikov function $M\left(I_{0}, \theta_{0}\right)$ at the resonance is given by the formula

$$
M\left(I_{0}, \theta_{0}\right)=H_{1}\left(X\left(I_{0}\right), I_{0}, \theta_{0}-\Delta \theta_{-}\right)-H_{1}\left(X\left(I_{0}\right), I_{0}, \theta_{0}+\Delta \theta_{+}\right) .
$$

PROOF: At the resonance, $D_{I} H_{0}\left(X\left(I_{0}\right), I_{0}\right)=0$, so for every heteroclinic orbit $a(t)$ with $I=I_{0}$, we have

$$
\mathbf{n}(a(t))=\left(D_{x} H_{0}(a(t)), D_{I} H_{0}(a(t)), 0\right),
$$


and

$$
\begin{aligned}
\langle\mathbf{n}(a(t)), \mathbf{g}(a(t))\rangle & =\left\langle D_{x} H_{0}(a(t)), J D_{x} H_{1}(a(t))\right\rangle-D_{I} H_{0}(a(t)) D_{\theta} H_{1}(a(t)) \\
& =-\left\langle J D_{x} H_{0}(a(t)), D_{x} H_{1}(a(t))\right\rangle-D_{I} H_{0}(a(t)) D_{\theta} H_{1}(a(t)) \\
& =-\left\langle\dot{x}(a(t)), D_{x} H_{1}(a(t))\right\rangle-\dot{\theta}(a(t)) D_{\theta} H_{1}(a(t)) \\
& =-\frac{d}{d t} H_{1}(a(t)),
\end{aligned}
$$

since $\dot{I}(a(t))=0$. Integration between $t=-\infty$ and $t=\infty$ now yields formula (4.3).

Now, since $H_{1}\left(X\left(I_{0}\right), I_{0}, \theta\right)$ is periodic in $\theta$ with period $2 \pi$,

$$
\int_{0}^{2 \pi} M\left(I_{0}, \theta_{0}\right) d \theta_{0}=0
$$

and therefore, if $M\left(I_{0}, \theta_{0}\right)$ is not identically equal to zero, it must pass through zero at least twice for $0 \leq \theta_{0} \leq 2 \pi$. (The number of zeros of $M\left(I_{0}, \theta_{0}\right)$, including their multiplicity, must be even). In general, we cannot say whether all the zeros of $M\left(I_{0}, \theta_{0}\right)$ will be simple. However, generically, $M\left(I_{0}, \theta_{0}\right)$ will have an even number of simple zeros.

An interesting nongeneric case occurs when $\Delta \theta$ is a multiple of $2 \pi$, that is, when the heteroclinic orbits connecting pairs of equilibria on the unperturbed resonant circle become homoclinic orbits, connecting those equilibria to themselves rather than to other equilibria. In this case, the Melnikov function at the resonance vanishes identically, and the existence of homoclinic intersections cannot be established. We will ignore this case in the rest of the paper.

As a corollary to the above discussion, we can state

Proposition 4.2 Generically, for all I close enough (but not equal) to $I_{0}$, the stable and unstable manifolds $W^{s}\left(O_{\varepsilon}^{I}\right)$ and $W^{u}\left(O_{\varepsilon}^{I}\right)$ of the periodic orbits $O_{\varepsilon}^{I}$ intersect transversely inside the corresponding level surface of the perturbed Hamiltonian $H$.

PROOF: Generically, $M\left(I_{0}, \bar{\theta}_{0}\right)=0$ and $D_{\theta_{0}} M\left(I_{0}, \bar{\theta}_{0}\right) \neq 0$ for some $\overline{\theta_{0}}$. Therefore, by the implicit function theorem, there exists a function $\bar{\theta}_{0}(I)$ with $\bar{\theta}_{0}\left(I_{0}\right)=\bar{\theta}_{0}$ such that $M\left(I, \bar{\theta}_{0}(I)\right)=0$ and $D_{\theta_{0}} M\left(I_{0}, \bar{\theta}_{0}(I)\right) \neq 0$ for all $I$ close enough to $I_{0}$. Hence, it follows that the manifolds $W^{s}\left(\mathcal{M}_{\varepsilon}\right)$ and $W^{u}\left(\mathcal{M}_{\varepsilon}\right)$ intersect along a two-dimensional intersection surface $\Sigma_{\varepsilon}\left(\bar{\theta}_{0}\right)$ for $I$ close enough to $I_{0}$. This surface is foliated by level surfaces of the perturbed 
Hamiltonian $H$ into orbits homoclinic to periodic orbits $O_{\varepsilon}^{I}$, that is, intersection orbits of the manifolds $W^{s}\left(O_{\varepsilon}^{I}\right)$ and $W^{u}\left(O_{\varepsilon}^{I}\right)$.

Now, away from $I=I_{0}$, the intersection surface $\Sigma_{\varepsilon}\left(\bar{\theta}_{0}\right)$ and the level surfaces of the Hamiltonian $H$ intersect transversely. This is because their unperturbed counterparts, the limiting surface $\Sigma\left(\bar{\theta}_{0}\right)$ and the unperturbed level surfaces of the Hamiltonian $H_{0}$, intersect transversely, which we prove below by showing that the tangent space of $\Sigma\left(\bar{\theta}_{0}\right)$ is not orthogonal to the normals of the level surfaces of the Hamiltonian $H_{0}$. (That the intersection surface $\Sigma_{\varepsilon}\left(\bar{\theta}_{0}\right)$ collapses smoothly onto the limiting surface $\Sigma\left(\bar{\theta}_{0}\right)$ is a consequence of proposition 3.4.)

To calculate two independent tangents of the limiting surface $\Sigma\left(\bar{\theta}_{0}\right)$, we note that it is parametrized by $t$ and $I$ in the equation

$$
\Sigma\left(\bar{\theta}_{0}\right)=\left\{(x, I, \theta) \mid(x, I, \theta)=\left(x^{h}(t, I), I, \theta^{h}\left(t, I, \bar{\theta}_{0}(I)\right)\right)\right\} .
$$

Therefore, its two independent tangents $\mathbf{t}_{t}$ and $\mathbf{t}_{I}$ are the partial derivatives of the expressions for $(x, I, \theta)$ with respect to $t$ and $I$, that is,

$$
\mathbf{t}_{t}=\left(J D_{x} H_{0}\left(x^{h}(t, I), I\right), 0, D_{I} H_{0}\left(x^{h}(t, I), I\right)\right),
$$

and

$$
\mathbf{t}_{I}=\left(D_{I} x^{h}(t, I), 1, D_{I} \theta^{h}\left(t, I, \bar{\theta}_{0}(I)\right)\right) .
$$

The normals to the level surfaces of the Hamiltonian $H_{0}$ are

$$
\mathbf{n}_{H_{0}}=\left(D_{x} H_{0}\left(x^{h}(t, I), I\right), D_{I} H_{0}\left(x^{h}(t, I), I\right), 0\right) .
$$

The scalar product $\left\langle\mathbf{t}_{t}, \mathbf{n}_{H_{0}}\right\rangle$ is clearly always zero; however, the scalar product $\left\langle\mathbf{t}_{I}, \mathbf{n}_{H_{0}}\right\rangle$ is equal to

$$
\left\langle\mathbf{t}_{I}, \mathbf{n}_{H_{0}}\right\rangle=\frac{d}{d I} H_{0}\left(x^{h}(t, I), I\right) .
$$

By differentiating equation (2.9) with respect to $I$, and taking into account that $X(I)$ is an equilibrium of $(2.3 \mathrm{a})$, we find that

$$
\left\langle\mathbf{t}_{I}, \mathbf{n}_{H_{0}}\right\rangle=D_{I} H_{0}(X(I), I),
$$

which is nonzero when $I \neq I_{0}$.

Thus, we have shown that, away from $I=I_{0}$, the intersection surface $\Sigma_{\varepsilon}\left(\bar{\theta}_{0}\right)$ and the level surfaces of the Hamiltonian $H$ intersect transversely, and that the manifolds $W^{s}\left(\mathcal{M}_{\varepsilon}\right)$ and $W^{u}\left(\mathcal{M}_{\varepsilon}\right)$ intersect transversely along the surface $\Sigma_{\varepsilon}\left(\bar{\theta}_{0}\right)$. Therefore, the intersections 
$W^{s}\left(O_{\varepsilon}^{I}\right)$ and $W^{u}\left(O_{\varepsilon}^{I}\right)$ of the manifolds $W^{s}\left(\mathcal{M}_{\varepsilon}\right)$ and $W^{u}\left(\mathcal{M}_{\varepsilon}\right)$ with the level surfaces of the Hamiltonian $H$ intersect each other transversely inside those level surfaces, and the proof is complete.

From proposition 4.2 it follows via the Smale-Birkhoff homoclinic theorem (see, for instance, Wiggins [1988]) that the intersecting manifolds $W^{s}\left(O_{\varepsilon}^{I}\right)$ and $W^{u}\left(O_{\varepsilon}^{I}\right)$ cause nearby dynamics to become chaotic. This is a well-known result (see Holmes and Marsden [1982], Robinson [1988], or Wiggins [1988]), and for the rest of this paper we will be concerned with how these chaotic dynamics change as we pass through the resonance at $I=I_{0}$.

\section{The Resonance Band}

We now turn our attention to the dynamics on the perturbed annulus $\mathcal{M}_{\varepsilon}$ near the resonance at $I=I_{0}$. For this purpose, we "blow up" the resonance by the substitution

$$
I=I_{0}+\sqrt{\varepsilon} h .
$$

The equations for $I$ and $\theta$ restricted to $\mathcal{M}_{\varepsilon}$ near $I=I_{0}$ yield the equations for $h$ and $\theta$ there, namely

$$
\begin{aligned}
\dot{h} & =-\sqrt{\varepsilon} D_{\theta} H_{1}\left(X\left(I_{0}\right), I_{0}, \theta\right)+\mathcal{O}(\varepsilon) \\
\dot{\theta} & =\sqrt{\varepsilon} \frac{d}{d I}\left[D_{I} H_{0}\left(X\left(I_{0}\right), I_{0}\right)\right] h+\mathcal{O}(\varepsilon),
\end{aligned}
$$

where we have expanded the right-hand side of (5.2) in powers of $\sqrt{\varepsilon}$ and used the resonance condition (2.6). When we introduce the slow time $\tau=\sqrt{\varepsilon} t$ and let $\varepsilon \rightarrow 0$, we find the limiting rescaled or outer system

$$
\begin{aligned}
h^{\prime} & =-D_{\theta} H_{1}\left(X\left(I_{0}\right), I_{0}, \theta\right) \\
\theta^{\prime} & =\frac{d}{d I}\left[D_{I} H_{0}\left(X\left(I_{0}\right), I_{0}\right)\right] h
\end{aligned}
$$

with $^{\prime}=\frac{d}{d \tau}$. This system can be derived from the rescaled Hamiltonian

$$
\mathcal{H}(h, \theta)=\frac{1}{2} \frac{d}{d I}\left[D_{I} H_{0}\left(X\left(I_{0}\right), I_{0}\right)\right] h^{2}+H_{1}\left(X\left(I_{0}\right), I_{0}, \theta\right)
$$

via the canonical formulas

$$
h^{\prime}=-D_{\theta} \mathcal{H}(h, \theta), \quad \theta^{\prime}=D_{h} \mathcal{H}(h, \theta)
$$

System (5.3) describes the limiting dynamics in the resonance band created by the perturbation out of the circle of equilibria at $I=I_{0}$. We remark that, when higher order terms 


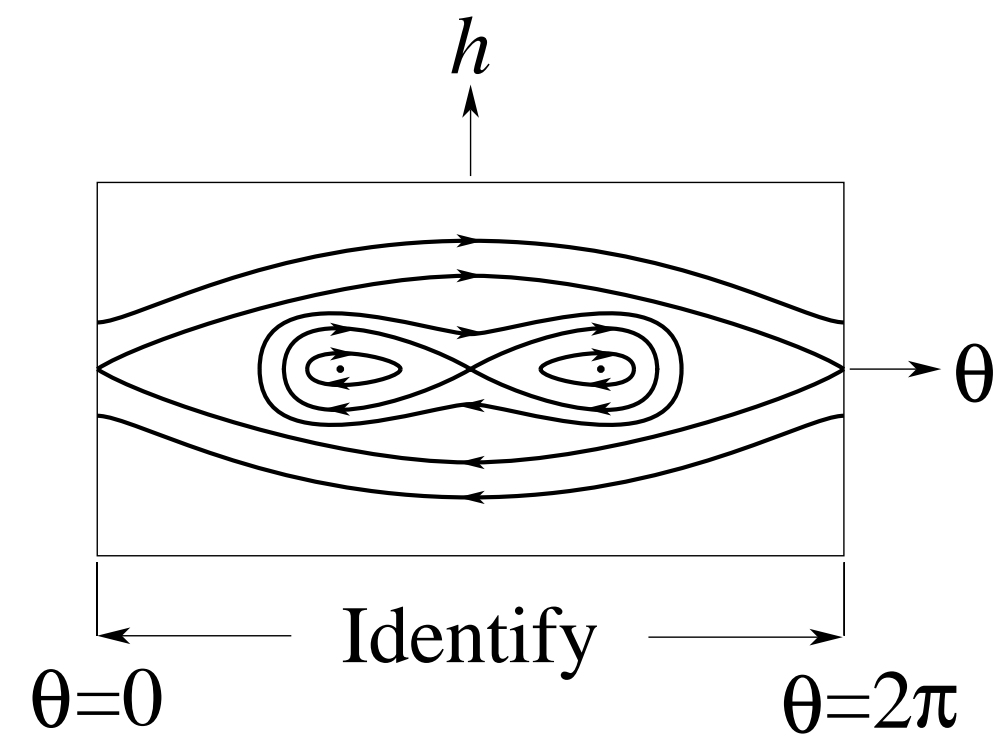

Figure 3: Dynamics on the $h-\theta$ cylinder in a resonance band.

are added to the equations (5.3), these equations need not have a canonical Hamiltonian structure any longer.

From its structure and the structure of the Hamiltonian (5.4), we see that system (5.3) is pendulum-like. Thus, we have

Proposition 5.1 The $h-\theta$ phase cylinder of equations (5.3) is symmetric with respect to reflections across the circle $h=0$. Equilibria of equations (5.3) must lie on the $\theta$-axis. Moreover, there is an equal number of stable and unstable equilibria, with neighboring equilibria being of opposite stability type.

The last part of this proposition is true because $H_{1}\left(X\left(I_{0}\right), I_{0}, \theta\right)$ is periodic in $\theta$ with period $2 \pi$, and because, by assumption 4 , all the zeros of $D_{\theta} H_{1}\left(X\left(I_{0}\right), I_{0}, \theta\right)$ are simple.

The unstable equilibria will, in general, be connected either to themselves, or to other unstable equilibria by homoclinic or heteroclinic orbits, forming separatrices on the $h-\theta$ cylinder. Stable equilibria will be surrounded by nested families of periodic orbits, which do not wind around the cylinder. For large values of $h$, orbits in the $h-\theta$ cylinder are periodic orbits that wind around the cylinder once. These periodic orbits correspond to the perturbed periodic orbits $O_{\varepsilon}^{I}$ that are close to the resonance. For a typical phase portrait in the resonance band, see figure 3 .

In order to describe the dynamics of the full equations (5.2) we note that the restriction 
$\left.H\right|_{\mathcal{M}_{\varepsilon}}(h, \theta)$ of the Hamiltonian $H(x, I, \theta, \varepsilon)$ to the perturbed invariant annulus $\mathcal{M}_{\varepsilon}$ is a constant of motion for the equations (5.2), even though it is not necessarily the canonical Hamiltonian for these equations. We expand this restricted function $\left.H\right|_{\mathcal{M}_{\varepsilon}}(h, \theta)$, namely, (cf. proposition 3.1)

$$
\begin{array}{r}
\left.H\right|_{\mathcal{M}_{\varepsilon}}(h, \theta)=H_{0}\left(X\left(I_{0}+\sqrt{\varepsilon} h\right)+\varepsilon X_{1}\left(I_{0}+\sqrt{\varepsilon} h, \theta\right)+\mathcal{O}\left(\varepsilon^{2}\right), I_{0}+\sqrt{\varepsilon} h\right) \\
+\varepsilon H_{1}\left(X\left(I_{0}+\sqrt{\varepsilon} h\right)+\varepsilon X_{1}\left(I_{0}+\sqrt{\varepsilon} h, \theta\right)+\mathcal{O}\left(\varepsilon^{2}\right), I_{0}+\sqrt{\varepsilon} h, \theta\right)
\end{array}
$$

in powers of $\sqrt{\varepsilon}$ and retain terms up to $\mathcal{O}(\varepsilon)$. The result of this expansion is

$$
\left.H\right|_{\mathcal{M}_{\varepsilon}}(h, \theta)=H_{0}\left(X\left(I_{0}\right), I_{0}\right)+\varepsilon\left[\frac{1}{2} \frac{d}{d I}\left[D_{I} H_{0}\left(X\left(I_{0}\right), I_{0}\right)\right] h^{2}+H_{1}\left(X\left(I_{0}\right), I_{0}, \theta\right)\right]+\mathcal{O}(\varepsilon \sqrt{\varepsilon}) .
$$

We thus conclude that the difference

$$
\mathcal{H}_{\varepsilon}(h, \theta)=\frac{\left.H\right|_{\mathcal{M}_{\varepsilon}}(h, \theta)-H_{0}\left(X\left(I_{0}\right), I_{0}\right)}{\varepsilon}=\mathcal{H}(h, \theta)+\mathcal{O}(\sqrt{\varepsilon})
$$

is a constant of motion for the equations (5.2), which reduces smoothly to the rescaled Hamiltonian $\mathcal{H}$ as $\sqrt{\varepsilon} \rightarrow 0$. Orbits and equilibria of the equations (5.2) are thus going to coincide with level curves and critical points of the constant $\mathcal{H}_{\varepsilon}(h, \theta)$.

The existence of the constant $\mathcal{H}_{\varepsilon}(h, \theta)$ implies

Proposition 5.2 For small positive $\varepsilon$, the number of equilibria and their stability does not change from the $\varepsilon=0$ case. If an equilibrium is connected to itself by a homoclinic orbit at $\varepsilon=0$, a unique homoclinic orbit persists $\mathcal{O}(\sqrt{\varepsilon})$ away (in $h-\theta$ coordinates) from the unperturbed one for small positive $\varepsilon$. If $O$ is a periodic orbit for $\varepsilon=0$ and $\mathcal{H}(h, \theta)=\mathcal{H}(O)$ on $O$, a unique periodic orbit $O_{\varepsilon}$ with $\mathcal{H}_{\varepsilon}(h, \theta)=\mathcal{H}(O)$ persists for small positive $\varepsilon$ a distance $\mathcal{O}(\sqrt{\varepsilon})$ away (in $h-\theta$ coordinates) from the periodic orbit $O$.

PROOF: Since all the zeros of $D_{\theta} H_{1}\left(X\left(I_{0}\right), I_{0}, \theta\right)$ are simple, the number of equilibria and their stability does not change when we add higher order terms to the Hamiltonian $\mathcal{H}(h, \theta)$ in the constant $\mathcal{H}_{\varepsilon}(h, \theta)$. Continuity and nonzero gradient of $\mathcal{H}_{\varepsilon}(h, \theta)$ imply persistence of homoclinic and periodic orbits, as well as guarantee their uniqueness.

The only structures that may not survive under perturbation are heteroclinic connections between pairs of equilibria. Those may break up and form pairs of homoclinic orbits, enclosing a new family of periodic orbits. Therefore, situations involving heteroclinic orbits must be dealt with on a case-by-case basis. However, if we exclude heteroclinic orbits and their 
$\mathcal{O}(\sqrt{\varepsilon})$ thin (in $h-\theta$ coordinates) neighborhoods from our consideration, the rest of the objects on the $h-\theta$ cylinder are structurally stable. Therefore, the dynamics on the perturbed annulus $\mathcal{M}_{\varepsilon}$ near the resonance at $I=I_{0}$ are approximately, and qualitatively correctly, described by the rescaled Hamiltonian $\mathcal{H}(h, \theta)$ and the corresponding outer equations (5.3).

From the substitutions we have performed, it should be evident that the resonant dynamics take place in an $\mathcal{O}(\sqrt{\varepsilon})$ thin (in $x-I-\theta$ coordinates) resonance band on slow, $t=\mathcal{O}\left(\frac{1}{\sqrt{\varepsilon}}\right)$, time scales.

\section{Geometric Singular Perturbation Theory}

In the next two sections we will concentrate our attention on how to couple the dynamics near the resonance on the perturbed annulus $\mathcal{M}_{\varepsilon}$ with the dynamics in the homoclinic intersections that we discussed in section 4 . To set the stage for this investigation, we recall the result given in proposition 4.2, namely, that, away from the resonance, two-dimensional intersection surfaces of the three-dimensional manifolds $W^{s}\left(\mathcal{M}_{\varepsilon}\right)$ and $W^{u}\left(\mathcal{M}_{\varepsilon}\right)$ are sliced by the three-dimensional surfaces of constant Hamiltonian $H$ into discrete intersection orbits of the two-dimensional stable and unstable manifolds $W^{s}\left(O_{\varepsilon}^{I}\right)$ and $W^{u}\left(O_{\varepsilon}^{I}\right)$ of the perturbed periodic orbits $O_{\varepsilon}^{I}$. The reason why this result is fairly simple is because, by proposition 3.4 , orbits on the perturbed manifolds, $W^{s}\left(O_{\varepsilon}^{I}\right)$ and $W^{u}\left(O_{\varepsilon}^{I}\right)$, are close to orbits on the unperturbed manifolds $W^{s}\left(O^{I}\right)$ and $W^{u}\left(O^{I}\right)$ over semi-infinite time intervals. That is, as the orbits approach the periodic orbit $O_{\varepsilon}^{I}$ along its stable or unstable manifold, their dynamics differ only slightly from the dynamics of the unperturbed orbits approaching the periodic orbit $O^{I}$ along its stable or unstable manifold. Hence, the problem that is discussed in proposition 4.2 is a regular perturbation problem.

The situation becomes much more complicated around the resonant value $I=I_{0}$. There, the unperturbed periodic orbit $O^{I_{0}}$ has an infinite period, that is, $O^{I_{0}}$ is a circle of equilibria. Its perturbed counterpart, however, is a resonance band, which is mostly filled with periodic orbits. Therefore, the unperturbed and the perturbed dynamics are drastically different, and, thus, we are dealing with a singular perturbation problem.

In order to bring out its singular perturbation structure, we rewrite the system (2.1) in the $x-h-\theta$ variables, namely,

$$
\dot{x}=J D_{x} H_{0}\left(x, I_{0}+\sqrt{\varepsilon} h\right)+\varepsilon J D_{x} H_{1}\left(x, I_{0}+\sqrt{\varepsilon} h, \theta\right),
$$




$$
\begin{gathered}
\dot{h}=-\sqrt{\varepsilon} D_{\theta} H_{1}\left(x, I_{0}+\sqrt{\varepsilon} h, \theta\right), \\
\dot{\theta}=D_{I} H_{0}\left(x, I_{0}+\sqrt{\varepsilon} h\right)+\varepsilon D_{I} H_{1}\left(x, I_{0}+\sqrt{\varepsilon} h, \theta\right),
\end{gathered}
$$

and consider two of its limits.

The first limit is obtained by restricting $x$ to lie on the perturbed manifold $\mathcal{M}_{\varepsilon}$ and rescaling time by $\sqrt{\varepsilon}$ to become the slow time $\tau=\sqrt{\varepsilon} t$. We then let $\varepsilon \rightarrow 0$. Not unexpectedly, the equations we thus obtain on $\mathcal{M}_{\varepsilon}$ are precisely the rescaled equations (5.3):

$$
\begin{aligned}
h^{\prime} & =-D_{\theta} H_{1}\left(X\left(I_{0}\right), I_{0}, \theta\right), \\
\theta^{\prime} & =\frac{d}{d I}\left[D_{I} H_{0}\left(X\left(I_{0}\right), I_{0}\right)\right] h,
\end{aligned}
$$

with $^{\prime}=\frac{d}{d \tau}$. This limit is called the outer limit of the equations (6.1).

The second relevant limit is obtained by simply setting $\varepsilon=0$ in the equations (6.1), and is called the inner limit of the equations (6.1). The equations we obtained this way are

$$
\begin{gathered}
\dot{x}=J D_{x} H_{0}\left(x, I_{0}\right), \\
\dot{h}=0, \\
\dot{\theta}=D_{I} H_{0}\left(x, I_{0}\right) .
\end{gathered}
$$

The manifold $\mathcal{M}_{\varepsilon}$ in this inner limit collapses smoothly onto the $h-\theta$ cylinder $\mathcal{M}_{0}$, which is the set

$$
\mathcal{M}_{0}=\left\{(x, h, \theta) \mid x=X\left(I_{0}\right)\right\}
$$

and we see that $\dot{h}=\dot{\theta}=0$ there. Hence, in the inner limit, the circle of equilibria at $I=I_{0}$ has been "blown up" into the whole $h-\theta$ cylinder $\mathcal{M}_{0}$. Thus

Proposition 6.1 The system (6.2) possesses a cylinder $\mathcal{M}_{0}$ of equilibria at $x=X\left(I_{0}\right)$, parametrized by $h$ and $\theta$. Equilibria on this cylinder are all unstable, and pairs of these equilibria that are $\Delta \theta$ apart are connected to each other by heteroclinic orbits parametrized by $t, h$ and $\theta_{0}$ in

$$
(x, h, \theta)=\left(x^{h}\left(t, I_{0}\right), h, \theta^{h}\left(t, I_{0}, \theta_{0}\right)\right)
$$


In order to couple the dynamics near the resonance on the perturbed annulus $\mathcal{M}_{\varepsilon}$ and the dynamics transverse to $\mathcal{M}_{\varepsilon}$, we use the two limiting systems of equations we have just discussed: The outer system (5.3) and the inner system (6.2). The cylinder $\mathcal{M}_{0}$ of equilibria at $x=X\left(I_{0}\right)$, parametrized by $h$ and $\theta$, is a normally hyperbolic invariant surface for the system (6.2). This surface persists together with its stable and unstable manifolds for nonzero $\varepsilon$ in the $x-h-\theta$ phase space of the equations (6.1) in the same way as described in section 3. Indeed, the persisting hyperbolic manifold is just $\mathcal{M}_{\varepsilon}$, represented in $x-h-\theta$ coordinates.

We can, however, go further in the description of the dynamics inside the manifolds $W^{s}\left(\mathcal{M}_{\varepsilon}\right)$ and $W^{u}\left(\mathcal{M}_{\varepsilon}\right)$ close to the resonant value $I=I_{0}$. In particular, theorem 9.1 in Fenichel [1979] applies to systems of the same type as (6.1), and guarantees that the local manifolds $W_{l o c}^{s}\left(\mathcal{M}_{\varepsilon}\right)$ and $W_{l o c}^{u}\left(\mathcal{M}_{\varepsilon}\right)$ are foliated by stable and unstable fibers. The statement of this theorem, which is tailored to the needs of the present paper, is given in the next proposition. The estimates in this proposition are stated in the $x-h-\theta$ coordinates.

Proposition 6.2 For all small enough $\varepsilon$, the local stable manifold $W_{\text {loc }}^{s}\left(\mathcal{M}_{\varepsilon}\right)$ of the invariant annulus $\mathcal{M}_{\varepsilon}$ is foliated by a family of disjoint curves called stable fibers, and the local unstable manifold $W_{\text {loc }}^{u}\left(\mathcal{M}_{\varepsilon}\right)$ of the invariant annulus $\mathcal{M}_{\varepsilon}$ is foliated by a family of disjoint curves called unstable fibers. Stable fibers form a positively invariant family, that is, the image, under the forward time flow of any stable fiber is contained in a stable fiber. Likewise, unstable fibers form a negatively invariant family, that is, the image, under the backward time flow of any unstable fiber is contained in an unstable fiber. Each fiber pierces the annulus $\mathcal{M}_{\varepsilon}$ transversely (inside the corresponding manifold $W_{\text {loc }}^{s}\left(\mathcal{M}_{\varepsilon}\right)$ or $W_{\text {loc }}^{u}\left(\mathcal{M}_{\varepsilon}\right)$ ) in precisely one point, called its base point. As the base points on $\mathcal{M}_{\varepsilon}$ move under the dynamics of the vector field (6.1), the stable and unstable fibers move along with their base points and contract exponentially towards their base points in forward and backward time, respectively. The exponential contraction rate for stable fibers is $e^{-\left[\lambda\left(I_{0}\right)+\mathcal{O}(\sqrt{\varepsilon})\right] t}$ as $t \rightarrow \infty$, and the exponential contraction rate for unstable fibers is $e^{\left[\lambda\left(I_{0}\right)+\mathcal{O}(\sqrt{\varepsilon})\right] t}$ as $t \rightarrow-\infty$, where $\lambda\left(I_{0}\right)$ is the absolute value of (both) the eigenvalues of the equation (2.3a) at $I=I_{0}$ (or, equivalently, the inner equation (6.2a)) linearized around $x=X\left(I_{0}\right)$. The families of stable and unstable fibers vary smoothly with $\sqrt{\varepsilon}$ and other parameters in the problem. For $\varepsilon=0$, that is, for system (6.2), the stable and unstable fibers are precisely the local stable and unstable manifolds of the equilibria on the $h-\theta$ cylinder $\mathcal{M}_{0}$.

Theorems similar to proposition 6.2 are also stated in Sakamoto [1990], Jones and Kopell 
[1994], and Kovačič and Wiggins [1992]. Proposition 6.2 implies that one can think of the stable and unstable fibers as "traveling" stable and unstable manifolds of their base points.

We here make a remark about the smoothness of the stable and unstable fibers: If the vector field (6.1) is $r$-times continuously differentiable, the fibers are $(r-1)$-times continuously differentiable. However, this remark is of little practical concern, since, in practice, most vector fields are analytic, and the corresponding stable and unstable fibers are as smooth as we please. (For a more detailed explanation of this issue, see Fenichel [1979].)

Fenichel's fibers enable us to construct local stable and unstable manifolds of objects on the annulus $\mathcal{M}_{\varepsilon}$ near the resonance:

Proposition 6.3 For every periodic orbit on the perturbed annulus $\mathcal{M}_{\varepsilon}$, its local stable manifold is the union of all the stable fibers whose base points lie on that orbit. For all the equilibria in the resonance band that are centers for the system (6.1) restricted to the annulus $\mathcal{M}_{\varepsilon}$, that is, equations (5.2), their local stable manifolds are precisely the fibers having these centers as their base points. For the equilibria that are saddles for the restricted system (5.2), the local parts of their stable manifolds that are near the annulus $\mathcal{M}_{\varepsilon}$ are the unions of the fibers with base points lying on the restricted stable manifolds of these saddles on the annulus $\mathcal{M}_{\varepsilon}$, whose dynamics are governed by equations (5.2). Similar statements hold for the local unstable manifolds of objects that lie on the annulus $\mathcal{M}_{\varepsilon}$.

We obtain the full global stable and unstable manifolds of orbits and equilibria that lie on the perturbed annulus $\mathcal{M}_{\varepsilon}$ by evolving their local counterparts in forward and backward time, respectively. We thus see that the three-dimensional stable and unstable manifolds $W^{s}\left(\mathcal{M}_{\varepsilon}\right)$ and $W^{u}\left(\mathcal{M}_{\varepsilon}\right)$ close to the resonance at $I=I_{0}$ are foliated for nonzero $\varepsilon$ by the two-dimensional stable and unstable manifolds of the periodic orbits in the resonance band, as well as the one-dimensional or two-dimensional stable and unstable manifolds of the equilibria.

We can obtain, using both systems of equations (5.3) and (6.2), a geometric limiting structure of the foliations of the perturbed annulus $\mathcal{M}_{\varepsilon}$ and its stable and unstable manifolds $W^{s}\left(\mathcal{M}_{\varepsilon}\right)$ and $W^{u}\left(\mathcal{M}_{\varepsilon}\right)$. In particular if $O_{\varepsilon}$ is a periodic orbit with $\mathcal{H}_{\varepsilon}\left(O_{\varepsilon}\right)=\mathcal{H}_{O}$, it will limit, as $\varepsilon \rightarrow 0$, to a closed curve $O$. This curve is a level curve of the rescaled Hamiltonian $\mathcal{H}(h, \theta)$ at the value $\mathcal{H}(h, \theta)=\mathcal{H}_{O}$. The closed curve $O$ is a periodic orbit for the outer equations (5.3), and a closed curve of equilibria for the inner equations (6.2). Propositions 6.2 and 6.3 now imply 

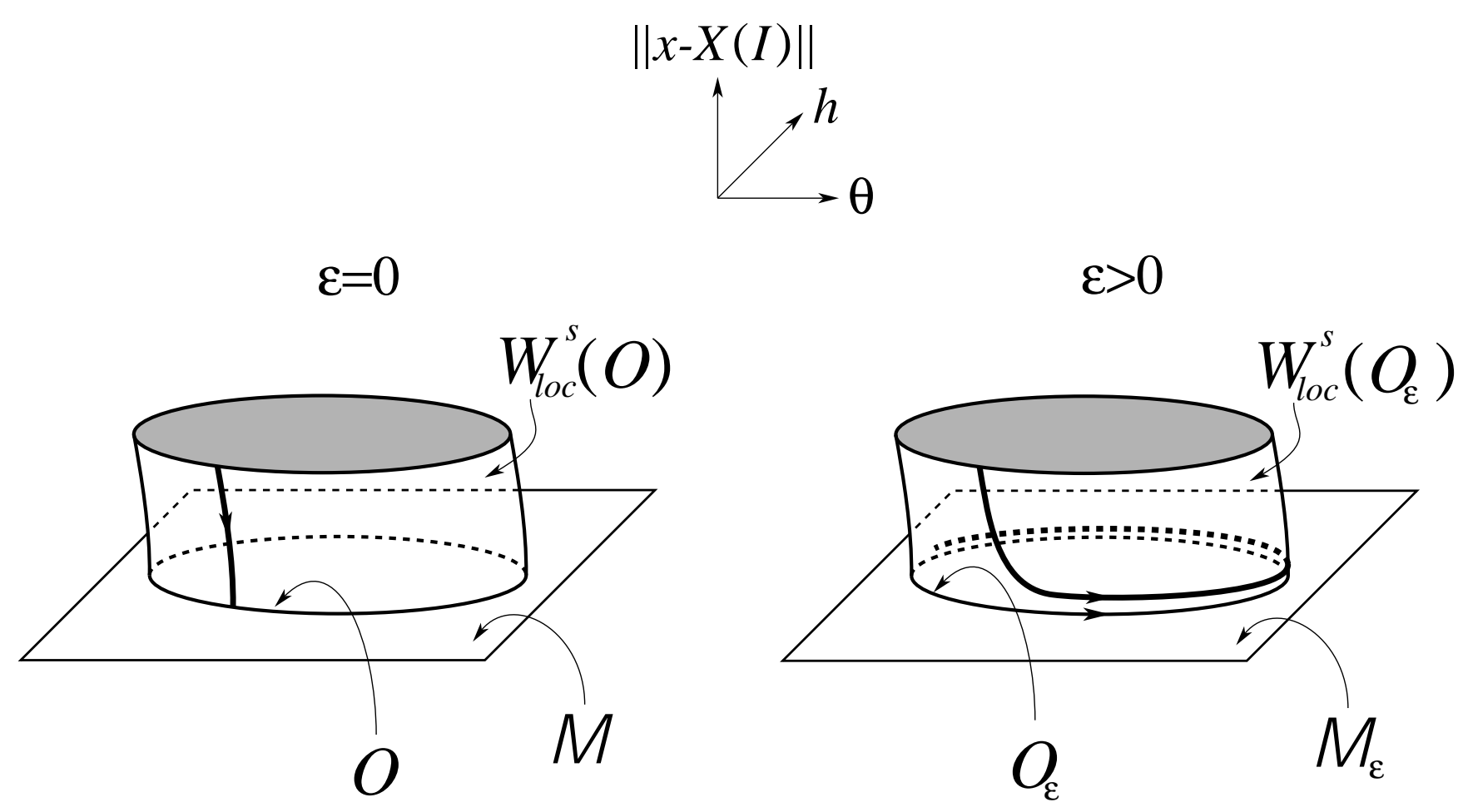

Figure 4: As $\varepsilon \rightarrow 0$, the local stable manifold, $W_{l o c}^{s}\left(O_{\varepsilon}\right)$, of the periodic orbit $O_{\varepsilon}$ limits on the union, $W_{l o c}^{s}(O)$, of the local stable manifolds of equilibria that lie on the limiting curve $O$ at $\varepsilon=0$.

Proposition 6.4 In the $x-h-\theta$ coordinates, the local stable and unstable manifolds of the periodic orbit $O_{\varepsilon}$ limit, as $\varepsilon \rightarrow 0$, onto the local stable and unstable manifolds of the curve $O$. These latter manifolds are the unions of the (one-dimensional) local stable and unstable manifolds of the equilibria (under the dynamics of the inner equations (6.2)) comprising the curve $O$. The local stable and unstable manifolds of the curves $O_{\varepsilon}$ and $O$ are $\mathcal{O}(\sqrt{\varepsilon})$ apart, respectively.

For an illustration of this proposition, see figure 4.

Similar results can be obtained for the limiting local stable and unstable manifolds of equilibria, including those that are connected to themselves by homoclinic orbits on $\mathcal{M}_{\varepsilon}$. 


\section{Orbits Homoclinic to a Resonance Band}

In this section we finally couple the dynamics near the resonance band in $\mathcal{M}_{\varepsilon}$ with the dynamics on the surviving homoclinic orbits as discussed in the section 4 .

Throughout this whole section we assume the situation that we considered at the end of section 4, namely

Assumption 5 The Melnikov function $M\left(I_{0}, \theta_{0}\right)$ has a simple zero at some $\theta_{0}=\bar{\theta}_{0}$, that is, $M\left(I_{0}, \bar{\theta}_{0}\right)=0$ with $D_{\theta_{0}} M\left(I_{0}, \bar{\theta}_{0}\right) \neq 0$.

Then, for some function $\bar{\theta}_{0}(I)$ and close enough to $I=I_{0}$, we have $M\left(I, \bar{\theta}_{0}(I)\right)=0$ and $D_{\theta_{0}} M\left(I, \bar{\theta}_{0}(I)\right) \neq 0$. Therefore, in this case the stable and unstable manifolds $W^{s}\left(\mathcal{M}_{\varepsilon}\right)$ and $W^{u}\left(\mathcal{M}_{\varepsilon}\right)$ of the perturbed annulus $\mathcal{M}_{\varepsilon}$ intersect transversely along a two-dimensional surface $\Sigma_{\varepsilon}\left(\bar{\theta}_{0}\right)$, parametrized by $I$ and $t$ with $\theta_{0}=\bar{\theta}_{0}(I)$.

We now set $I=I_{0}+\sqrt{\varepsilon} h$, and let $\varepsilon \rightarrow 0$. We denote the limit (in $x-h-\theta$ space) as $\varepsilon$ tends to zero of the intersection surface $\Sigma_{\varepsilon}\left(\bar{\theta}_{0}\right)$ by $\Sigma_{0}\left(\bar{\theta}_{0}\right)$. Proposition 6.1 now implies

Proposition 7.1 The limiting surface $\Sigma_{0}\left(\bar{\theta}_{0}\right)$ consists of those heteroclinic orbits connecting equilibria on the $h-\theta$ cylinder $\mathcal{M}_{0}$ (under the dynamics of the inner equations (6.2)) whose $\theta_{0}$ parameter equals $\theta_{0}=\bar{\theta}_{0}\left(I_{0}\right)=\bar{\theta}_{0}$. These orbits are thus explicitly given by the formula

$$
(x, h, \theta)=\left(x^{h}\left(t, I_{0}\right), h, \theta^{h}\left(t, I_{0}, \bar{\theta}_{0}\right)\right) .
$$

Heteroclinic orbits comprising the surface $\Sigma_{0}\left(\bar{\theta}_{0}\right)$ emerge from the $h-\theta$ cylinder $\mathcal{M}_{0}$ along the line $\theta=\bar{\theta}_{0}-\Delta \theta_{-}$and return to $\mathcal{M}_{0}$ along the line $\theta=\bar{\theta}_{0}+\Delta \theta_{+}$.

An illustration of the limiting homoclinic surface $\Sigma_{0}\left(\bar{\theta}_{0}\right)$ is shown in figure 5 . Note that because of formulas (4.3) and (5.4), the end points of any heteroclinic orbit in $\Sigma_{0}\left(\bar{\theta}_{0}\right)$ possess the same value of the rescaled Hamiltonian $\mathcal{H}(h, \theta)$.

Typically, we may expect the lines $\theta=\bar{\theta}_{0}-\Delta \theta_{-}$and $\theta=\bar{\theta}_{0}+\Delta \theta_{+}$to cut (transversely) through nested families of periodic orbits of the outer system (5.3) on the cylinder $\mathcal{M}_{0}$. We therefore assume

Assumption 6 The line $\theta=\bar{\theta}_{0}-\Delta \theta_{-}$intersects transversely a periodic orbit, $O_{1}$, at $h=h_{0}$, and the line $\theta=\bar{\theta}_{0}+\Delta \theta_{+}$intersects transversely another periodic orbit, $\mathrm{O}_{2}$, at the same value $h=h_{0}$. 

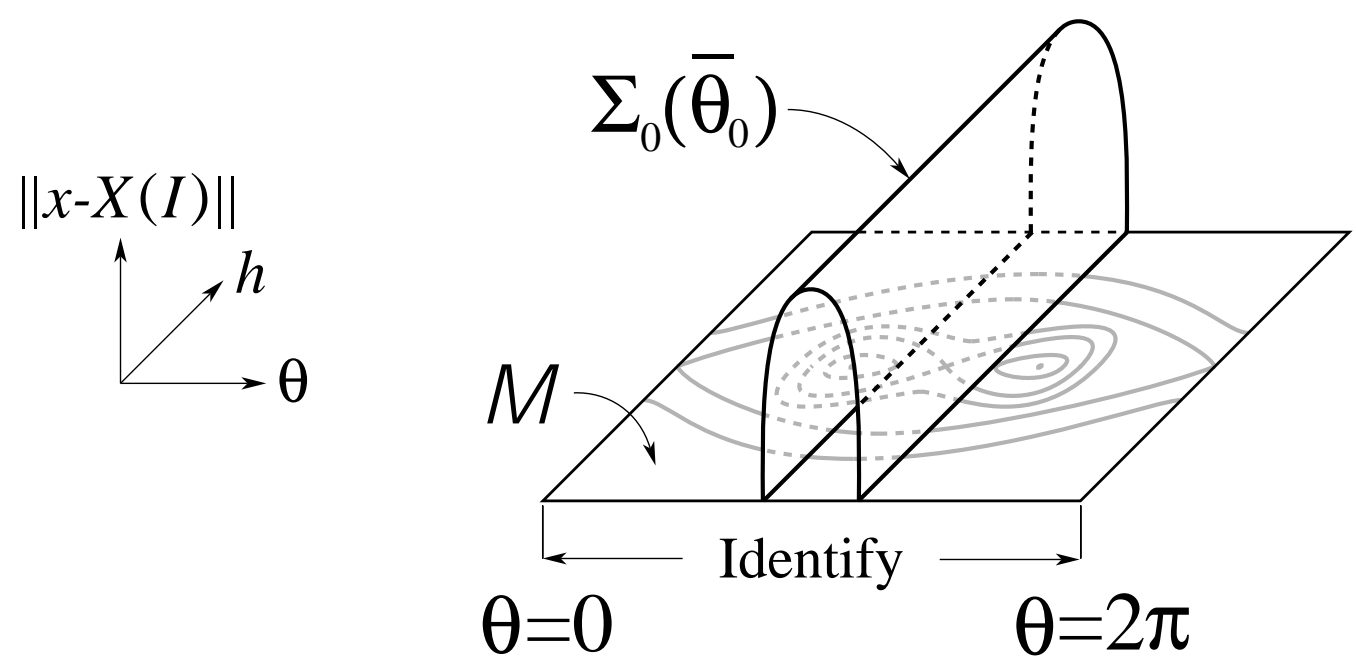

Figure 5: Geometry of the limiting intersection surface $\Sigma_{0}\left(\bar{\theta}_{0}\right)$.

Thus, the two points $\left(h_{0}, \bar{\theta}_{0}-\Delta \theta_{-}\right)$and $\left(h_{0}, \bar{\theta}_{0}+\Delta \theta_{+}\right)$are connected by one of the heteroclinic orbits forming the limiting surface $\Sigma_{0}\left(\bar{\theta}_{0}\right)$, namely the orbit denoted $a_{0}(t)$ parametrized by $(x, h, \theta)=\left(x^{h}\left(t, I_{0}\right), h_{0}, \theta^{h}\left(t, I_{0}, \bar{\theta}_{0}\right)\right)$. Note that the value of the rescaled Hamiltonian $\mathcal{H}(h, \theta)$ must be the same on both closed curves, $O_{1}$ and $O_{2}$. In many instances, the curves $\mathrm{O}_{1}$ and $\mathrm{O}_{2}$ coincide, $\mathrm{O}_{1}=\mathrm{O}_{2}$.

Let $\delta$ be a small positive number, independent of $\varepsilon$. An important preliminary result is

Proposition 7.2 The local part of the surface $\Sigma_{0}\left(\bar{\theta}_{0}\right)$ near the line $\theta=\bar{\theta}_{0}-\Delta \theta_{-}$(that is, the part contained in a $\delta$-neighborhood of this line) intersects the local unstable manifold $W_{\text {loc }}^{u}\left(O_{1}\right)$ of the curve $O_{1}$ along the heteroclinic orbit $a_{0}(t)$ transversely inside the local unstable manifold $W_{\text {loc }}^{u}\left(\mathcal{M}_{0}\right)$ of the $h-\theta$ cylinder $\mathcal{M}_{0}$. Likewise, the local part of the surface $\Sigma_{0}\left(\bar{\theta}_{0}\right)$ near the line $\theta=\bar{\theta}_{0}+\Delta \theta_{+}$intersects the local stable manifold $W_{\text {loc }}^{\text {s }}\left(O_{1}\right)$ of the curve $\mathrm{O}_{2}$ along the heteroclinic orbit $a_{0}(t)$ transversely inside the local stable manifold $W_{\text {loc }}^{s}\left(\mathcal{M}_{0}\right)$ of the $h-\theta$ cylinder $\mathcal{M}_{0}$.

This is because the local unstable manifolds of the line $\theta=\bar{\theta}_{0}-\Delta \theta_{-}$, of the orbit $O_{1}$, and of the cylinder $\mathcal{M}_{0}$, are just parallel translates of these objects along the unperturbed homoclinic orbits $\left(x^{h}\left(t, I_{0}\right), h, \theta^{h}\left(t, I_{0}, \theta_{0}\right)\right)$.

By proposition 5.2, for small nonzero $\varepsilon$, the curves $O_{1}$ and $O_{2}$ persist on $\mathcal{M}_{\varepsilon}$ as two perturbed periodic orbits, $O_{1, \varepsilon}$ and $O_{2, \varepsilon}$. Recall that $O_{1, \varepsilon}$ and $O_{2, \varepsilon}$ are those two perturbed periodic orbits on which the constant of motion $\mathcal{H}_{\varepsilon}(h, \theta)$ assumes the same value as $\mathcal{H}(h, \theta)$ 
assumes on the curves $O_{1}$ and $O_{2}$. (In $x-h-\theta$ coordinates, the curves $O_{1, \varepsilon}$ and $O_{1}$, and $O_{2, \varepsilon}$ and $O_{2}$ are $\mathcal{O}(\sqrt{\varepsilon})$ apart.) It is clear that the Hamiltonian $H(x, I, \theta, \varepsilon)$ must assume the same value on both orbits $O_{1, \varepsilon}$ and $O_{2, \varepsilon}$. We are now in the position to prove

Proposition 7.3 There exists a heteroclinic orbit connecting the perturbed periodic orbits $O_{1, \varepsilon}$ and $O_{2, \varepsilon}$. As $\varepsilon \rightarrow 0$, this heteroclinic orbit collapses onto the union of the curves $O_{1}$ and $\mathrm{O}_{2}$ and the orbit $a_{0}(t)$.

PROOF: We first show that the surfaces $\Sigma_{\varepsilon}\left(\bar{\theta}_{0}\right)$ and $W^{u}\left(O_{1, \varepsilon}\right)$ intersect along an orbit. We then show that this orbit must, in forward time, asymptote to an object on the perturbed cylinder $\mathcal{M}_{\varepsilon}$, and that this object is precisely the periodic orbit $O_{2, \varepsilon}$.

In order to show the existence of an intersection between the two surfaces $\Sigma_{\varepsilon}\left(\bar{\theta}_{0}\right)$ and $W^{u}\left(O_{1, \varepsilon}\right)$, first note that both the intersection surface $\Sigma_{\varepsilon}\left(\bar{\theta}_{0}\right)$ and the local unstable manifold $W_{l o c}^{u}\left(O_{1, \varepsilon}\right)$ of the periodic orbit $O_{1, \varepsilon}$, are contained in the unstable manifold $W^{u}\left(\mathcal{M}_{\varepsilon}\right)$. For $\frac{\delta}{2}<\left\|x-X\left(I_{0}\right)\right\|<\delta$, a proposition analogous to proposition 3.4 implies that the intersection surface $\Sigma_{\varepsilon}\left(\bar{\theta}_{0}\right)$ is $\mathcal{O}(\sqrt{\varepsilon})$ close to its limit $\Sigma_{0}\left(\bar{\theta}_{0}\right)$. Proposition 6.4 implies that the local unstable manifold $W_{l o c}^{u}\left(O_{1, \varepsilon}\right)$ is $\mathcal{O}(\sqrt{\varepsilon})$ close to its limit $W_{l o c}^{u}\left(O_{1}\right)$ in the $x-h-\theta$ coordinates. Moreover, by proposition 7.2, for $\frac{\delta}{2}<\left\|x-X\left(I_{0}\right)\right\|<\delta$, the part of the surface $\Sigma_{0}\left(\bar{\theta}_{0}\right)$ near the line $\theta=\bar{\theta}_{0}-\Delta \theta_{-}$intersects the local unstable manifold $W_{l o c}^{u}\left(O_{1}\right)$ of the curve $O_{1}$ transversely inside the local unstable manifold $W_{\text {loc }}^{u}\left(\mathcal{M}_{0}\right)$ of the $h-\theta$ cylinder $\mathcal{M}_{0}$. Therefore, by persistence of transverse intersections, the piece of the intersection surface $\Sigma_{\varepsilon}\left(\bar{\theta}_{0}\right)$ and the piece of the perturbed local unstable manifold $W_{\text {loc }}^{u}\left(O_{1, \varepsilon}\right)$ contained in $\frac{\delta}{2}<\left\|x-X\left(I_{0}\right)\right\|<\delta$ intersect transversely inside the perturbed unstable manifold $W^{u}\left(\mathcal{M}_{\varepsilon}\right)$. Because both the intersection surface $\Sigma_{\varepsilon}\left(\bar{\theta}_{0}\right)$ and the unstable manifold $W^{u}\left(O_{1, \varepsilon}\right)$ are invariant, they must intersect along a segment of an orbit, and this orbit, call it $a_{\varepsilon}(t)$, must in fact be contained in both $\Sigma_{\varepsilon}\left(\bar{\theta}_{0}\right)$ and $W^{u}\left(O_{1, \varepsilon}\right)$ for all times. A proposition analogous to proposition 3.4 implies that the trajectory $a_{\varepsilon}(t)$ must be $\mathcal{O}(\sqrt{\varepsilon})$ close to the trajectory $a_{0}(t)$ for all times $t$ with $-T \leq t \leq T$ and any positive $T$.

Now, since the manifolds $W^{s}\left(\mathcal{M}_{\varepsilon}\right)$ and $W^{u}\left(\mathcal{M}_{\varepsilon}\right)$ both contain the intersection surface $\Sigma_{\varepsilon}\left(\bar{\theta}_{0}\right)$, the orbit $a_{\varepsilon}(t)$ must asymptote in forward time to an object on the perturbed cylinder $\mathcal{M}_{\varepsilon}$. Since the trajectory $a_{\varepsilon}(t)$ must be $\mathcal{O}(\sqrt{\varepsilon})$ close to the trajectory $a_{0}(t)$ for all times $t$ with $-T \leq t \leq T$ and any positive $T$, we see that $a_{\varepsilon}(t)$ must pass through a $\delta$-neighborhood of the point $(x, h, \theta)=\left(X\left(I_{0}\right), h_{0}, \theta_{0}+\Delta \theta_{+}\right)$. By proposition 6.4, it follows that $a_{\varepsilon}(t)$ must pass at most $\mathcal{O}(\sqrt{\varepsilon})$ away from the local stable manifold $W_{\text {loc }}^{s}\left(O_{2, \varepsilon}\right)$ of the periodic orbit $O_{2, \varepsilon}$. But, for small enough $\varepsilon$, the local stable manifold $W_{l o c}^{s}\left(O_{2, \varepsilon}\right)$ is the only local stable 


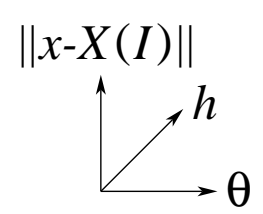

$\varepsilon=0$

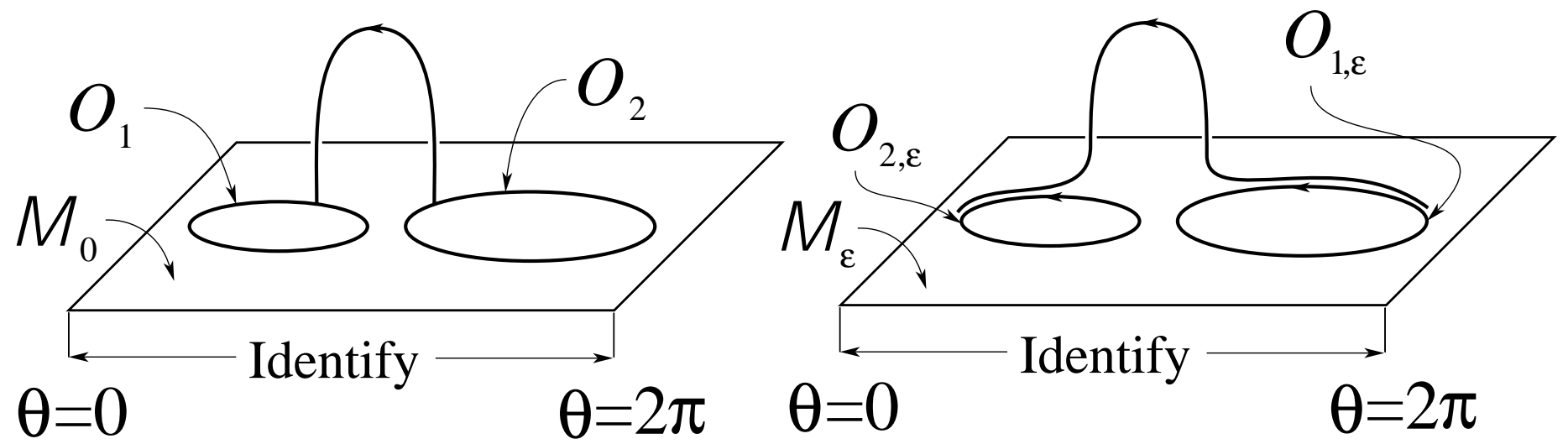

Figure 6: For small positive $\varepsilon$, a heteroclinic orbit connecting two periodic orbits, $O_{1, \varepsilon}$ and $\mathrm{O}_{2, \varepsilon}$, is born out of the limiting curves, $O_{1}$ and $O_{2}$, and the heteroclinic orbit on the limiting surface $\Sigma_{0}\left(\bar{\theta}_{0}\right)$ that connects an equilibrium on $O_{1}$ to an equilibrium on $O_{2}$ at $\varepsilon=0$.

manifold of an object on $\mathcal{M}_{\varepsilon}$ that is both $\mathcal{O}(\sqrt{\varepsilon})$ close to $W_{\text {loc }}^{s}\left(O_{2, \varepsilon}\right)$, and assumes the same value of the Hamiltonian $H(x, I, \theta, \varepsilon)$ as the periodic orbit $O_{1, \varepsilon}$. Therefore, the heteroclinic orbit $a_{\varepsilon}(t)$ must in fact enter the local stable manifold $W_{l o c}^{s}\left(O_{2, \varepsilon}\right)$ of the periodic orbit $O_{2, \varepsilon}$.

It should be clear from what we have just proven that, as $\varepsilon \rightarrow 0$, the heteroclinic orbit $a_{\varepsilon}(t)$ collapses onto the union of the curves $O_{1}$ and $O_{2}$ and the orbit $a_{0}(t)$. This concludes our proof.

In other words, proposition 7.3 states that surfaces of constant $H$ slice the intersection surface $\Sigma_{\varepsilon}\left(\bar{\theta}_{0}\right)$ into isolated heteroclinic or homoclinic orbits, connecting pairs of periodic orbits on the perturbed annulus $\mathcal{M}_{\varepsilon}$ to each other or periodic orbits on $\mathcal{M}_{\varepsilon}$ to themselves. An illustration of proposition 7.3 is shown in figure 6 .

It remains to answer whether the intersection of the stable manifold $W^{s}\left(O_{2, \varepsilon}\right)$ and the unstable manifold $W^{u}\left(O_{1, \varepsilon}\right)$ is transverse inside the corresponding level surface of the Hamiltonian $H$. The answer is provided by

Proposition 7.4 If the height $h_{0}$ at which the line $\theta=\bar{\theta}_{0}-\Delta \theta_{-}$intersects the periodic orbit $O_{1}$, and the line $\theta=\bar{\theta}_{0}+\Delta \theta_{+}$intersects the periodic orbit $O_{2}$, is nonzero, $h_{0} \neq 0$, then the 
intersection of the stable manifold $W^{s}\left(O_{2, \varepsilon}\right)$ and the unstable manifold $W^{u}\left(O_{1, \varepsilon}\right)$ along the heteroclinic orbit $a_{\varepsilon}(t)$ is transverse inside the corresponding level surface of the Hamiltonian $H$.

PROOF: This proof is a retracing of the argument given in the proof of proposition 4.2. Since the zero at $\theta_{0}=\bar{\theta}_{0}$ of the Melnikov function $M\left(I_{0}, \theta_{0}\right)$ corresponding to the homoclinic intersection surface $\Sigma_{\varepsilon}\left(\bar{\theta}_{0}\right)$ is simple, the manifolds $W^{s}\left(\mathcal{M}_{\varepsilon}\right)$ and $W^{u}\left(\mathcal{M}_{\varepsilon}\right)$ intersect transversely at every point of that intersection surface. Moreover, by the discussion folowing proposition 6.3, the manifolds $W^{s}\left(\mathcal{M}_{\varepsilon}\right)$ and $W^{u}\left(\mathcal{M}_{\varepsilon}\right)$ are foliated by surfaces of constant Hamiltonian $H$ into the stable and unstable manifolds of objects on the annulus $\mathcal{M}_{\varepsilon}$. In particular, it follows that since the manifolds $W^{s}\left(\mathcal{M}_{\varepsilon}\right)$ and $W^{u}\left(\mathcal{M}_{\varepsilon}\right)$ pass through each other with nonzero speed along their intersection surface $\Sigma_{\varepsilon}\left(\bar{\theta}_{0}\right)$ as $\theta_{0}$ passes through $\theta_{0}=\bar{\theta}_{0}$, the manifolds $W^{s}\left(O_{2, \varepsilon}\right)$ and $W^{u}\left(O_{1, \varepsilon}\right)$ must also pass through each other with nonzero speed (along an isolated intersection orbit) inside the corresponding surface of constant Hamiltonian $H$, provided that this surface and the intersection surface $\Sigma_{\varepsilon}\left(\bar{\theta}_{0}\right)$ intersect transversely.

We thus proceed to show that, when $h_{0} \neq 0$, the level surfaces of the Hamiltonian $H$ and the intersection surface $\Sigma_{\varepsilon}\left(\bar{\theta}_{0}\right)$ indeed intersect transversely. In the proof of proposition 4.2, we showed that, in the $x-I-\theta$ coordinates, the projection $\left\langle\mathbf{t}_{I}, \mathbf{n}_{H_{0}}\right\rangle$ of the normal $\mathbf{n}_{H_{0}}$ to the level surfaces of the Hamiltonian $H_{0}$ onto the tangent space of the intersection surface $\Sigma\left(\bar{\theta}_{0}\right)$ is given by the expression $D_{I} H_{0}(X(I), I)$. For nonzero $\varepsilon$, the size of the analogous projection at any given point changes by an $\mathcal{O}(\varepsilon)$ amount, as can be shown from the definition of the surfaces $\Sigma\left(\bar{\theta}_{0}\right)$ and $\Sigma_{\varepsilon}\left(\bar{\theta}_{0}\right)$, and propositions 3.2 and 3.4. Thus, for $I=I_{0}+\sqrt{\varepsilon} h_{0}+\mathcal{O}(\varepsilon)$, the projection of the normal to a level surface of the Hamiltonian $H$ onto the tangent space of the intersection surface $\Sigma_{\varepsilon}\left(\bar{\theta}_{0}\right)$ is equal to $\sqrt{\varepsilon} \frac{d}{d I} D_{I} H_{0}\left(X\left(I_{0}\right), I_{0}\right) h_{0}+\mathcal{O}(\varepsilon)$; that is, this projection can pass through zero only when $h_{0}$ does, which proves our proposition.

Incidentally, the preceeding proof shows that, in the $x-I-\theta$ coordinates, the size of the angle between the intersecting manifolds $W^{s}\left(O_{2, \varepsilon}\right)$ and $W^{u}\left(O_{1, \varepsilon}\right)$ inside the corresponding level surface of the Hamiltonian $H$ is $\mathcal{O}(\varepsilon \sqrt{\varepsilon})$. Namely, the Melnikov function calculation described in section 4 immediately implies that the size of the angle between the intersecting manifolds $W^{s}\left(\mathcal{M}_{\varepsilon}\right)$ and $W^{u}\left(\mathcal{M}_{\varepsilon}\right)$ is $\mathcal{O}(\varepsilon)$, and we have just shown that the size of the angle between the intersection surface $\Sigma_{\varepsilon}\left(\bar{\theta}_{0}\right)$ and the level surfaces of the Hamiltonian $H$ is $\mathcal{O}(\sqrt{\varepsilon})$, because this angle is proportional to the perturbed projection, $\left\langle\mathbf{t}_{I}, \mathbf{n}_{H_{0}}\right\rangle+\mathcal{O}(\varepsilon)$.

All the preceeding propositions from this section can now be summarized in the Main Theorem Let for a system of the form (2.1) the assumptions 1 through 4 be satisfied. 
Let $\theta_{0}=\bar{\theta}_{0}$ be a solution of the equation

$$
H_{1}\left(X\left(I_{0}\right), I_{0}, \theta_{0}-\Delta \theta_{-}\right)-H_{1}\left(X\left(I_{0}\right), I_{0}, \theta_{0}+\Delta \theta_{+}\right)=0
$$

with

$$
D_{\theta_{0}} H_{1}\left(X\left(I_{0}\right), I_{0}, \bar{\theta}_{0}-\Delta \theta_{-}\right)-D_{\theta_{0}} H_{1}\left(X\left(I_{0}\right), I_{0}, \bar{\theta}_{0}+\Delta \theta_{+}\right) \neq 0 .
$$

Furthermore, let the lines $\theta=\bar{\theta}_{0}-\Delta \theta_{-}$and $\theta=\bar{\theta}_{0}+\Delta \theta_{+}$intersect two periodic orbits, $O_{1}$ and $\mathrm{O}_{2}$, of the outer system (5.3) transversely on the $h-\theta$ cylinder $\mathcal{M}_{0}$, both at the same height $h=h_{0} \neq 0$. Then, for sufficiently small nonzero $\varepsilon$, there exist two periodic orbits $O_{1, \varepsilon}$ and $O_{2, \varepsilon}$ on the perturbed annulus $\mathcal{M}_{\varepsilon}$, which are $\mathcal{O}(\sqrt{\varepsilon})$ close to the curves $O_{1}$ and $O_{2}$ in the $x-h-\theta$ coordinates, respectively, and satisfy the condition $\mathcal{H}_{\varepsilon}\left(O_{1, \varepsilon}\right)=\mathcal{H}_{\varepsilon}\left(O_{2, \varepsilon}\right)=$ $\mathcal{H}\left(O_{1}\right)=\mathcal{H}\left(O_{2}\right)$. Moreover, the unstable manifold $W^{u}\left(O_{1, \varepsilon}\right)$ of the orbit $O_{1, \varepsilon}$ intersects the stable manifold $W^{s}\left(O_{2, \varepsilon}\right)$ of the orbit $O_{2, \varepsilon}$ transversely inside the corresponding level surface of the Hamiltonian $H(x, I, \theta, \varepsilon)$.

Remarks made after proposition 5.1 show that for all large enough $h_{0}$, the lines $\theta=$ $\bar{\theta}_{0}-\Delta \theta_{-}$and $\theta=\bar{\theta}_{0}+\Delta \theta_{+}$must intersect the same periodic orbit of the outer system (5.3) at $h=h_{0}$, that is, $O_{1}=O_{2}$. This is the situation that is described in proposition 4.2. Thus, the Main Theorem provides for a smooth transition between orbits homoclinic to the resonance band and orbits homoclinic to periodic orbits outside the resonance band.

We remark that only slight modifications of this theorem are needed in the cases when $O_{1}$ is the unstable manifold of a saddle on the annulus $\mathcal{M}_{0}$ (under the dynamics of the outer system (5.3)), or when $\mathrm{O}_{2}$ is the stable manifold of a saddle on $\mathcal{M}_{0}$.

Situations in which the Main Theorem applies often lead to the presence of chaotic dynamics near the intersection orbits of the manifolds $W^{u}\left(O_{1, \varepsilon}\right)$ and $W^{s}\left(O_{2, \varepsilon}\right)$. In particular, if the periodic orbits $O_{2, \varepsilon}$ and $O_{1, \varepsilon}$ coincide, $O_{2, \varepsilon}=O_{1, \varepsilon}$, a Poincaré map may be set up by fixing the phase along this periodic orbit, and transverse intersections of the stable and unstable manifolds of this orbit imply, via the Smale-Birkhoff homoclinic theorem, the existence of Smale-horseshoe-like chaotic dynamics for this Poincaré map.

Likewise, suppose two distinct orbits $O_{2, \varepsilon}$ and $O_{1, \varepsilon}$ are connected by a heteroclinic cycle consisting of (at least) two heteroclinic orbits, the trajectories on one heteroclinic orbit moving from $O_{1, \varepsilon}$ to $O_{2, \varepsilon}$ and the trajectories on another heteroclinic orbit returning from $O_{1, \varepsilon}$ to $O_{2, \varepsilon}$. Then, transverse intersections of the manifolds $W^{s}\left(O_{2, \varepsilon}\right)$ and $W^{u}\left(O_{1, \varepsilon}\right)$ and $W^{u}\left(O_{2, \varepsilon}\right)$ and $W^{s}\left(O_{1, \varepsilon}\right)$, respectively, along the two heteroclinic orbits imply existence of chaotic dynamics as well. (See, for instance, Guckenheimer and Holmes [1983].) 
To conclude this section, we discuss the necessity of assumptions 1 through 4 . Of these assumptions, 1 and 3 are necessary, because they define the class of problems of orbits homoclinic to resonance bands. Assumption 2, the twist condition, is less crucial. First, this twist condition can be relaxed to hold only at the resonance, $I=I_{0}$. This weakened assumption alone would ensure the existence of an $I$-interval (possibly smaller than $I_{1} \leq I \leq$ $I_{2}$ ) in which the twist condition is satisfied. However, we can omit assumption 2 entirely, as long as we assume that a derivative $\frac{d^{n}}{d I^{n}} D_{I} H_{0}\left(x\left(I_{0}\right), I_{0}\right)$ is nonzero for some finite $n$. In this case, the rescaling of the resonance is different, but the final result would be very similar. And finally, assumption 4 eliminates special cases in which equilibria in the resonance band undergo a bifurcation. In case a zero of $D_{\theta} H_{1}\left(x\left(I_{0}\right), I_{0}, \theta_{0}\right)$ is not simple, we only need to exclude an $\mathcal{O}(\sqrt{\varepsilon})$ neighborhood (in $x-h-\theta$ coordinates) of the corresponding equilibrium of the outer equations (5.3) from the investigation. This exclusion only affects proposition 5.2 , and has no influence on either the statement or the proof of the Main Theorem. With all this said, however, notice that assumptions 2 and 4 hold for generic problems and, hence, can be thought of as genericity assumptions.

\section{An Example}

In this section we present an example of the theory developed in the preceeding sections. We consider a one-parameter family of problems in which an ideal Duffing oscillator is coupled to an anharmonic oscillator, described by the Hamiltonian

$$
\begin{aligned}
H(p, q, I, \theta) & =H_{0}(p, q, I)+\varepsilon H_{1}(p, q, I, \theta) \\
& =\frac{1}{2} I^{2}-I+\frac{1}{2} p^{2}-\frac{1}{2} \mu^{2} q^{2}\left(I-\frac{1}{2} q^{2}\right)-\varepsilon I \cos \theta .
\end{aligned}
$$

Here, $\mu$ is a positive parameter. (See also Kovačič [1992a].)

At $\varepsilon=0$, the equations derived from this Hamiltonian are

$$
\begin{gathered}
\dot{p}=\mu^{2} q\left(I-\frac{1}{2} q^{2}\right) \\
\dot{q}=p \\
\dot{I}=0 \\
\dot{\theta}=I-1-\frac{1}{2} \mu^{2} q^{2} .
\end{gathered}
$$

Simple phase-plane analysis reveals that for all positive $I$ the planar system (8.2) possesses an unstable equilibrium at the origin $(p, q)=(0,0)$ which is connected to itself by a symmetric 


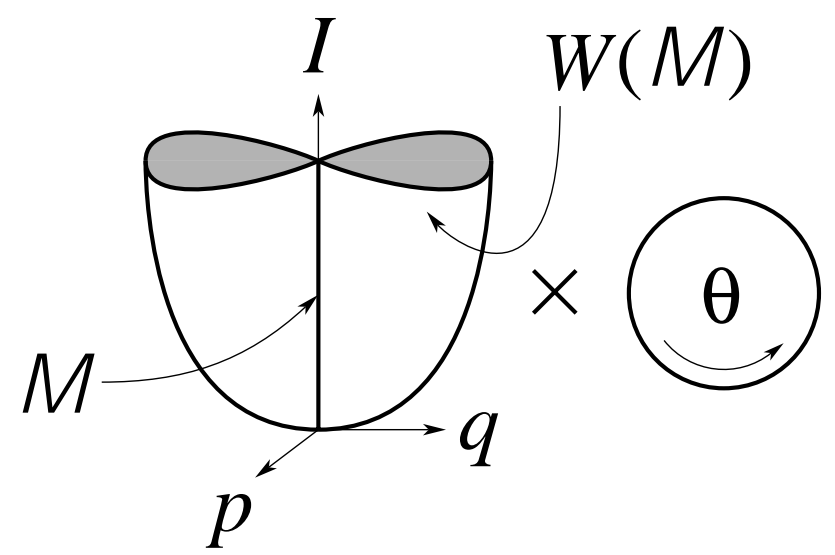

Figure 7: Geometry of the invariant annulus $\mathcal{M}$ and its two-branched homoclinic manifold $W(\mathcal{M})$ in the Duffing oscillator-anharmonic oscillator example.

pair of separatrices. Thus, we can take the normally hyperbolic annulus $\mathcal{M}$ to be any annulus with coordinates $p=q=0,0<I_{1}<I<I_{2}, 0 \leq \theta \leq 2 \pi$ for any positive $I_{1}$ and $I_{2}$, with $I_{1}<I_{2}$. The angular motion on $\mathcal{M}$ is given by the equation $\theta=(I-1) t+\theta_{0}$. The twist condition $\frac{d}{d I} D_{I} H_{0}(p, q, I)=1 \neq 0$ is fulfilled on $\mathcal{M}$ and there is a resonant value at $I=1$. Therefore, we choose $I_{1}$, and $I_{2}$ to satisfy the inequality $0<I_{1}<1<I_{2}$.

The unperturbed solutions on the orbits homoclinic to the annulus $\mathcal{M}$ are easily shown to be

$$
\begin{gathered}
p=\mp \sqrt{2} \mu I \operatorname{sech}(\mu \sqrt{I} t) \tanh (\mu \sqrt{I} t) \\
q= \pm \sqrt{2 I} \operatorname{sech}(\mu \sqrt{I} t) \\
I=I \\
\theta=(I-1) t-\mu \sqrt{I} \tanh (\mu \sqrt{I} t)+\theta_{0} .
\end{gathered}
$$

We immediately conclude that the phase difference $\Delta \theta$ at the resonance $I=1$ is equal to $\Delta \theta=-2 \mu$, and that $\Delta \theta_{-}=\Delta \theta_{+}=\frac{1}{2} \Delta \theta=-\mu$. Parameters $t, I$ and $\theta_{0}$ in the solutions (8.3) parametrize the two-branched homoclinic manifold $W(\mathcal{M})$, shown in figure 7 . One branch is described by the solution (8.3) with positive $q$, and the other branch is described by the solution (8.3) with negative $q$.

The $I-\theta$ cylinder at $p=q=0$ is invariant also for the perturbed problem. Results from section 5 imply that the rescaled Hamiltonian $\mathcal{H}(h, \theta)$ on this cylinder near the resonance is equal to

$$
\mathcal{H}(h, \theta)=\frac{1}{2} h^{2}-\cos \theta
$$


which is the pendulum Hamiltonian. The corresponding outer equations are

$$
\dot{h}=-\sin \theta, \quad \dot{\theta}=h .
$$

The features of the $h-\theta$ phase cylinder $\mathcal{M}_{0}$ of these equations are well known. In particular, there are two equilibria on this phase cylinder, a center at $(h, \theta)=(0,0)$ and a saddle at $(h, \theta)=(0, \pi)$. The saddle is connected to itself by two (homoclinic) separatrices that enclose a family of periodic orbits nested around the center. These periodic orbits are called libration orbits. The two families of periodic orbits above and below the libration region enclosed by the two separatrices wind once around the cylinder $\mathcal{M}_{0}$. They are called the rotation orbits. (The names libration and rotation come from the mechanical motions of the pendulum; see Goldstein [1980].)

All the features of the $h-\theta$ phase cylinder $\mathcal{M}_{0}$, including the two separatrices are structurally stable and survive the addition of higher order terms in $\varepsilon$ to the constant $\mathcal{H}(h, \theta)$. (In particular, this can be explicitly verified since the full perturbed resonant equations on $p=q=0$ can be derived from the Hamiltonian $\mathcal{H}_{\varepsilon}(h, \theta)=\frac{1}{2} h^{2}-(1+\sqrt{\varepsilon} h) \cos \theta$. $)$ By choosing two perturbed periodic orbits (one near $I=I_{1}$ and the other near $I=I_{2}$ ) as its boundaries, we can define the perturbed annulus $\mathcal{M}_{\varepsilon}$ as the part of the $I-\theta$ cylinder between these two orbits. Results from section 3 then imply the existence of its stable and unstable manifolds, $W^{s}\left(\mathcal{M}_{\varepsilon}\right)$ and $W^{u}\left(\mathcal{M}_{\varepsilon}\right)$.

The Melnikov function at the resonance is the same for both branches of the homoclinic manifold $W(\mathcal{M})$ and is equal to

$$
\begin{aligned}
M\left(1, \theta_{0}\right) & =\cos \left(\theta_{0}+\Delta \theta_{+}\right)-\cos \left(\theta_{0}-\Delta \theta_{-}\right) \\
& =\cos \left(\theta_{0}-\mu\right)-\cos \left(\theta_{0}+\mu\right) \\
& =2 \sin \mu \sin \theta_{0}
\end{aligned}
$$

When $\mu$ is not an integer multiple of $\pi$, the Melnikov function has simple zeros at $\theta_{0}=0$ and $\theta_{0}=\pi$. Thus the stable and unstable manifolds $W^{s}\left(\mathcal{M}_{\varepsilon}\right)$ and $W^{u}\left(\mathcal{M}_{\varepsilon}\right)$ intersect along two symmetric pairs of homoclinic surfaces, $\Sigma_{\varepsilon}^{ \pm}(0)$ and $\Sigma_{\varepsilon}^{ \pm}(\pi)$. In the $p-q-h-\theta$ coordinates, the two symmetric pairs of homoclinic surfaces, $\Sigma_{\varepsilon}^{ \pm}(0)$ and $\Sigma_{\varepsilon}^{ \pm}(\pi)$, collapse smoothly onto the two pairs of surfaces $\Sigma_{0}^{ \pm}(0)$ and $\Sigma_{0}^{ \pm}(\pi)$, parametrized by the expressions (8.3) with $I=1$, $\theta_{0}=0$ or $\theta_{0}=\pi$, and arbitrary constant $h$. The surfaces $\Sigma_{0}^{+}(0)$ and $\Sigma_{0}^{+}(\pi)$ are shown in figure 8 .

If $\mu>\pi$, the limiting homoclinic surfaces, $\Sigma_{0}^{ \pm}(0)$ and $\Sigma_{0}^{ \pm}(\pi)$, are hard to visualize. For $0<\mu<\pi$, however, we can visualize them fairly easily and by doing so discover in 


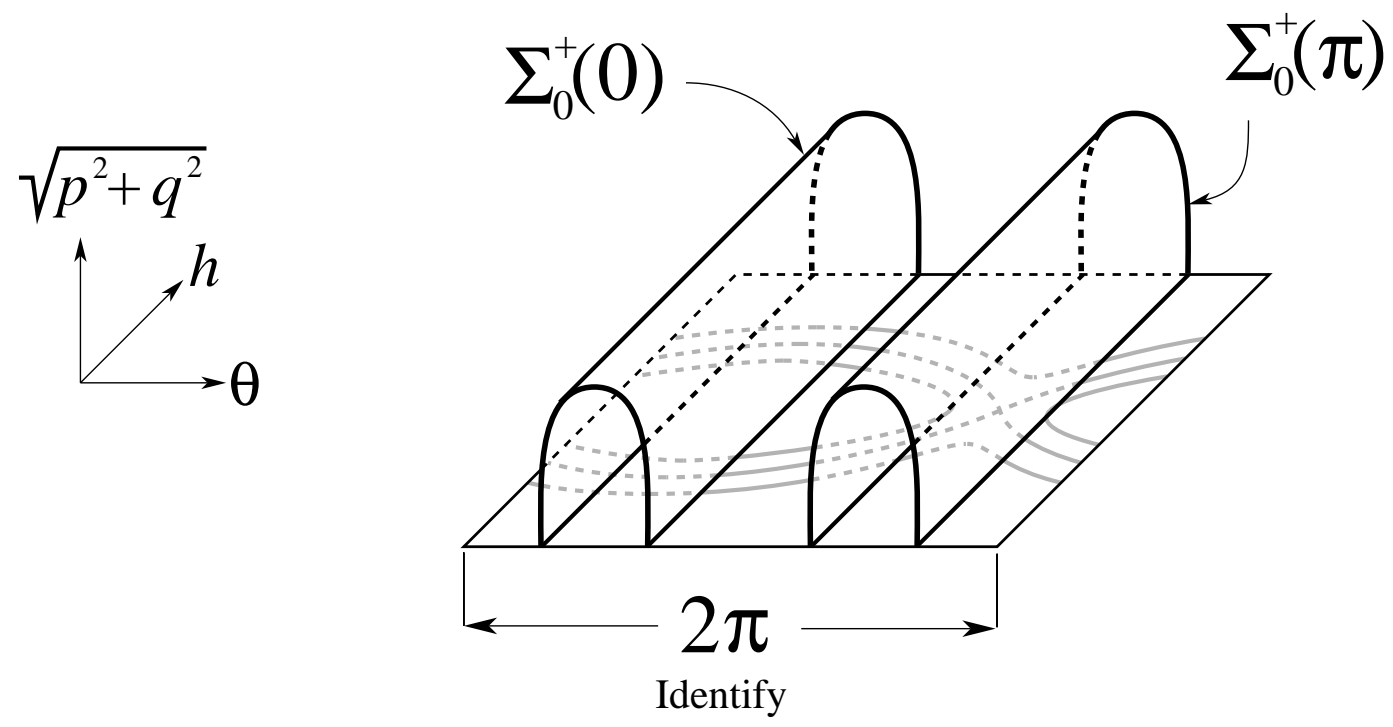

Figure 8: The two limiting intersection surfaces, $\Sigma_{0}^{+}(0)$ and $\Sigma_{0}^{+}(\pi)$.

this example the rich homoclinic structure that is implied by the Main Theorem. The two limiting intersection surfaces $\Sigma_{0}^{ \pm}(0)$ intersect the $h-\theta$ cylinder $\mathcal{M}_{0}$ along the lines $\theta=-\mu$ and $\theta=\mu$, and the two limiting intersection surfaces $\Sigma_{0}^{ \pm}(\pi)$ intersect the $h-\theta$ cylinder $\mathcal{M}_{0}$ along the lines $\theta=\pi-\mu$ and $\theta=\pi+\mu \equiv-\pi+\mu$. All the libration orbits of the equations (8.5) that extend beyond $\theta=-\mu$ and $\theta=\mu$ intersect these two lines transversely inside $\mathcal{M}_{0}$. Thus, each of these libration orbits is connected to itself by two pairs of heteroclinic orbits on $\Sigma_{0}^{ \pm}(0)$ : one at $h$ and another at $-h$, see figure 9. Each of the libration orbits that extend beyond $\theta=-\pi+\mu$ and $\theta=\pi-\mu$ is connected to itself by two pairs of heteroclinic orbits on $\Sigma_{0}^{ \pm}(\pi)$. In particular, if $\kappa=\max \{\mu, \pi-\mu\}$, each libration orbit that extends beyond $\theta=-\kappa$ and $\theta=\kappa$ is connected to itself by four pairs of homoclinic orbits, two pairs at $h$ and two pairs at $-h$. Each of the rotation orbits is connected to itself by one pair of heteroclinic orbits lying on the surfaces $\Sigma_{0}^{ \pm}(0)$, and by one pair of heteroclinic orbits lying on the surfaces $\Sigma_{0}^{ \pm}(\pi)$.

Chaotic dynamics can be proven to result from the existence of transverse homoclinic orbits in the current example, including the orbits homoclinic to the libration and rotation orbits in the resonance band. This can be shown by constructing a suitable Poincaré map near these periodic orbits. This construction is standard, and will not be carried out here. 

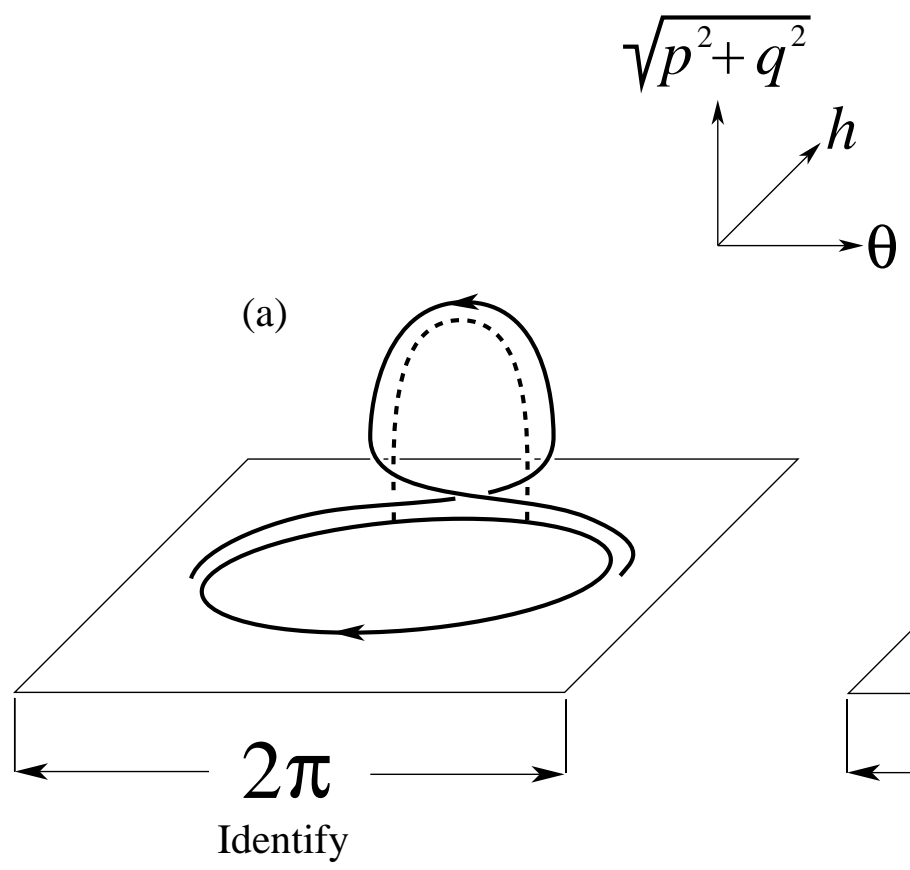

(b)

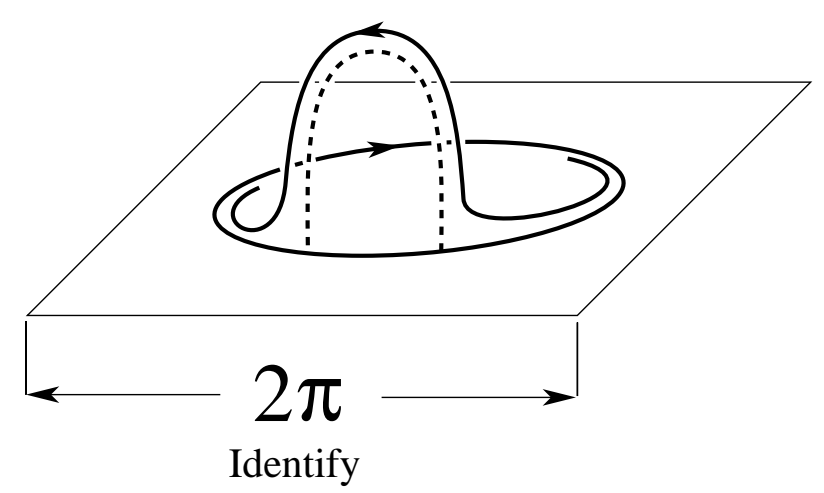

Figure 9: A perturbed homoclinic orbit that limits on the union of an unperturbed homoclinic orbit and a libration curve as $\varepsilon \rightarrow 0$ in the example in section 8. The case when $h>0$ is shown in figure $9 \mathrm{a}$, and the case when $h<0$ is shown in figure $9 \mathrm{~b}$.

\section{Extension to Several Degrees of Freedom}

In this section we outline an extension of the theory developed in the preceeding sections to the case when $x \in \mathbf{R}^{2 n}$ instead of $x \in \mathbf{R}^{2}$. This extension is rather straight forward. Therefore, we only point out the main differences between the two cases.

In section 2, equation (2.3a) retains its form, however, $J$ is now the matrix

$$
J=\left(\begin{array}{cc}
0 & -I d \\
I d & 0
\end{array}\right),
$$

where 0 is the $n \times n$ zero matrix, and $I d$ is the $n \times n$ identity matrix. In addition, we must assume

Assumption 7 There exist $n$ functions, $H_{0}(x, I)=K_{1}(x, I), \ldots, K_{n}(x, I)$, such that the gradients $D_{x} K_{1}(x, I), \ldots, D_{x} K_{n}(x, I)$ are pointwise linearly independent (except at the equilibria of (2.3a), where the gradient of the Hamiltonian vanishes), and whose Poisson brackets, $\left\{K_{i}(x, I), K_{j}(x, I)\right\}=\left\langle D_{x} K_{i}(x, I), J D_{x} K_{j}(x, I)\right\rangle$, all vanish.

The functions $K_{1}(x, I), \ldots, K_{n}(x, I)$ are constants of motion for the system (2.3a). 
Assumption 7 implies that equation (2.3a) is completely integrable. This complete integrability forces assumption 2 to change (see Wiggins [1988]), so that the equilibrium of the system (2.3a) at $x=X(I)$ is now connected to itself by an $(n-1)$-parameter family of homoclinic orbits $x^{h}(t, I, \phi)$, with $\phi \in \mathbf{R}^{n-1}$.

The unperturbed annulus $\mathcal{M}$ is defined exactly as in section 2 , but its stable and unstable manifolds are now $(n+2)$-dimensional. Two of their branches coincide to form an $(n+2)$ dimensional homoclinic manifold $W(\mathcal{M})$, parametrized by $t, I, \phi$, and $\theta_{0}$ in the homoclinic solutions

$$
\left(x^{h}(t, I, \phi), I, \theta^{h}\left(t, I, \theta_{0}\right)\right)
$$

with

$$
\theta\left(t, I, \theta_{0}\right)=\int_{0}^{t} D_{I} H_{0}\left(x^{h}(s, I, \phi), I\right) d s+\theta_{0} .
$$

At first, it may seem that the angle $\theta$ would have to depend on the $n-1$ parameters $\phi$, as well. However,

$$
\begin{aligned}
D_{\phi} \theta\left(t, I, \theta_{0}\right) & =\int_{0}^{t} D_{\phi} D_{I} H_{0}\left(x^{h}(s, I, \phi), I\right) d s \\
& =\int_{0}^{t} D_{I}\left[D_{\phi} H_{0}\left(x^{h}(s, I, \phi), I\right)\right] d s,
\end{aligned}
$$

and $D_{\phi} H_{0}\left(x^{h}(s, I, \phi), I\right)=0$ since $H_{0}\left(x^{h}(s, I, \phi), I\right)$ is constant for all $t$ and $\phi$, when $I$ is kept constant.

Alternatively, the homoclinic manifold $W(\mathcal{M})$ is described implicitly by the equations

$$
K_{i}(x, I)-K_{i}(X(I), I)=0, \quad i=1, \ldots, n .
$$

The resonant circle of equilibria at $I=I_{0}$ is connected to itself by an $(n+1)$-dimensional homoclinic manifold. This manifold is foliated by $n$-dimensional heteroclinic manifolds connecting pairs of equilibria that are

$$
\Delta \theta=\int_{-\infty}^{\infty} D_{I} H_{0}\left(x^{h}(s, I, \phi), I\right) d s
$$

away from each other.

All the results from section 3 now carry over to the general case with obvious modifications, and the results from section 5 carry over unchanged. The results from section 6 carry over with the slight modification that stable and unstable fibers are now $n$-dimensional. The only results that need to be modified are the results from section 4 . Namely, the homoclinic 
manifold $W(\mathcal{M})$ now has an $n$-dimensional normal space, and therefore an $n$-component Melnikov vector must be used instead of the one-component Melnikov function (see Wiggins [1988]). If this vector has a transverse zero, the results from section 7 readily carry over to the $n$-dimensional case.

We illustrate this extension in the example of the perturbed vector Duffing oscillatoranharmonic oscillator system, where $n=2$. This system is

$$
\begin{gathered}
\dot{p}_{1}=\mu^{2} q_{1}\left[I-\frac{1}{2}\left(q_{1}^{2}+q_{2}^{2}\right)\right]-\varepsilon \mu^{2} q_{1} \cos \theta \\
\dot{p}_{2}=\mu^{2} q_{2}\left[I-\frac{1}{2}\left(q_{1}^{2}+q_{2}^{2}\right)\right] \\
\dot{q}_{1}=p_{1} \\
\dot{q}_{2}=p_{2} \\
\dot{I}=-\varepsilon\left(I-\frac{1}{2} \mu^{2} q_{1}^{2}\right) \sin \theta \\
\dot{\theta}=I-1-\frac{1}{2} \mu^{2}\left(q_{1}^{2}+q_{2}^{2}\right)-\varepsilon \cos \theta
\end{gathered}
$$

Equations (9.1) are derived from the Hamiltonian

$$
\begin{aligned}
H\left(p_{1}, p_{2}, q_{1}, q_{2}, I, \theta\right)= & H_{0}\left(p_{1}, p_{2}, q_{1}, q_{2}, I\right)+\varepsilon H_{1}\left(p_{1}, p_{2}, q_{1}, q_{2}, I, \theta\right) \\
= & \frac{1}{2} I^{2}-I+\frac{1}{2}\left(p_{1}^{2}+p_{2}^{2}\right)-\frac{1}{2} \mu^{2}\left(q_{1}^{2}+q_{2}^{2}\right)\left[I-\frac{1}{2}\left(q_{1}^{2}+q_{2}^{2}\right)\right] \\
& -\varepsilon\left(I-\frac{1}{2} q_{1}^{2}\right) \cos \theta
\end{aligned}
$$

via the formulas

$$
\begin{gathered}
\dot{q}_{i}=\frac{\partial H\left(p_{1}, p_{2}, q_{1}, q_{2}, I, \theta\right)}{\partial p_{i}}, \quad \dot{p}_{i}=-\frac{\partial H\left(p_{1}, p_{2}, q_{1}, q_{2}, I, \theta\right)}{\partial q_{i}}, \quad i=1,2 \\
\dot{I}=-\frac{\partial H\left(p_{1}, p_{2}, q_{1}, q_{2}, I, \theta\right)}{\partial \theta}, \quad \dot{\theta}=\frac{\partial H\left(p_{1}, p_{2}, q_{1}, q_{2}, I, \theta\right)}{\partial I} .
\end{gathered}
$$

For $\varepsilon=0$, equations (9.1a) through (9.1d) are a one-parameter family of two-degreeof-freedom, completely integrable Hamiltonian systems, because they possess the additional constant of motion,

$$
K\left(p_{1}, p_{2}, q_{1}, q_{2}\right)=p_{1} q_{2}-p_{2} q_{1}
$$

For each positive $I$, the origin $p_{1}=p_{2}=q_{1}=q_{2}=0$ in (9.1a) through (9.1d) is a hyperbolic equilibrium with two positive and two negative eigenvalues. One can show (see Holm, Kovačič 
and Sundaram [1991]) that this equilibrium is connected to itself by a one-parameter family of homoclinic orbits

$$
\begin{array}{cl}
p_{1}=-\sqrt{2} \mu I \operatorname{sech}(\mu \sqrt{I} t) \tanh (\mu \sqrt{I} t) \cos \phi_{0}, \quad p_{2}=-\sqrt{2} \mu I \operatorname{sech}(\mu \sqrt{I} t) \tanh (\mu \sqrt{I} t) \sin \phi_{0}, \\
q_{1}=\sqrt{2 I} \operatorname{sech}(\mu \sqrt{I} t) \cos \phi_{0}, \quad q_{2}=\sqrt{2 I} \operatorname{sech}(\mu \sqrt{I} t) \sin \phi_{0},
\end{array}
$$

the parameter being $\phi_{0}$.

In the full phase space, the origin $p_{1}=p_{2}=q_{1}=q_{2}=0$ corresponds to an invariant cylinder, foliated by periodic orbits

$$
p_{1}=0, \quad p_{2}=0, \quad q_{1}=0, \quad q_{2}=0, \quad I=I, \quad \theta=(I-1) t+\theta_{0}
$$

Clearly the twist condition (2.5) is satisfied on the whole cylinder, and there is a resonance at $I=1$. The half-cylinder with $I>0$ is normally hyperbolic. We choose $I_{1}$ and $I_{2}$ so that $0<I_{1}<1<I_{2}$ and define the annulus $\mathcal{M}$ to be the part of the $I-\theta$ cylinder between $I=I_{1}$ and $I=I_{2}$.

The annulus $\mathcal{M}$ is normally hyperbolic and is connected to itself by a four-dimensional homoclinic manifold $W(\mathcal{M})$, parametrized by $t, I, \phi_{0}$ and $\theta_{0}$ in the formulas

$$
\begin{array}{crr}
p_{1}=-\sqrt{2} \mu I \operatorname{sech}(\mu \sqrt{I} t) \tanh (\mu \sqrt{I} t) \cos \phi_{0}, \quad & p_{2}=-\sqrt{2} \mu I \operatorname{sech}(\mu \sqrt{I} t) \tanh (\mu \sqrt{I} t) \sin \phi_{0}, \\
q_{1}=\sqrt{2 I} \operatorname{sech}(\mu \sqrt{I} t) \cos \phi_{0}, \quad q_{2}=\sqrt{2 I} \operatorname{sech}(\mu \sqrt{I} t) \sin \phi_{0}, & (9.3 \mathrm{a}) \\
I=I, \quad \theta=(I-1) t-\mu \sqrt{I} \tanh (\mu \sqrt{I} t)+\theta_{0} . & (9.3 \mathrm{c})
\end{array}
$$

At the resonance, (9.3c) implies that $\Delta \theta=-2 \mu$, and $\Delta \theta_{-}=\Delta \theta_{+}=\frac{1}{2} \Delta \theta=-\mu$, where $\Delta \theta_{-}$and $\Delta \theta_{+}$are defined as in section 4. Alternatively, the homoclinic manifold $W(\mathcal{M})$ is described implicitly by the equations

$$
H_{0}\left(p_{1}, p_{2}, q_{1}, q_{2}, I\right)-\frac{1}{2} I^{2}+I=0, \quad K\left(p_{1}, p_{2}, q_{1}, q_{2}\right)=0 .
$$

As in section 8, the perturbation leaves the $I-\theta$ cylinder at $p_{1}=p_{2}=q_{1}=q_{2}=0$ invariant. As mentioned above, results from sections 3 and 5 apply here exactly as in section 8, and the rescaled Hamiltonian and the outer equations are again (8.4) and (8.5). Results from section 6 apply here as well, except that stable and unstable fibers are now two-dimensional, instead of one-dimensional.

The results from section 4 must be modified, because the normal space of the homoclinic manifold $W(\mathcal{M})$ is now two-dimensional instead of one-dimensional. Therefore, a 
two-component Melnikov vector $\mathbf{M}\left(I, \phi_{0}, \theta_{0}\right)=\left(M_{1}\left(I, \phi_{0}, \theta_{0}\right), M_{2}\left(I, \phi_{0}, \theta_{0}\right)\right)$ must be used to determine the intersections of the perturbed stable and unstable manifolds $W^{s}\left(\mathcal{M}_{\varepsilon}\right)$ and $W^{u}\left(\mathcal{M}_{\varepsilon}\right)$ The two components of the Melnikov vector are proportional to the projections of the distance between the manifolds $W^{s}\left(\mathcal{M}_{\varepsilon}\right)$ and $W^{u}\left(\mathcal{M}_{\varepsilon}\right)$ near a given point $a \in W(\mathcal{M})$ onto the two normals to $W(\mathcal{M})$, namely, the gradients of the equations (9.4) that are given by

$$
\mathbf{n}_{1}(a)=\left(\frac{\partial H_{0}}{\partial p_{1}}(a), \frac{\partial H_{0}}{\partial p_{2}}(a), \frac{\partial H_{0}}{\partial q_{1}}(a), \frac{\partial H_{0}}{\partial q_{2}}(a), \frac{\partial H_{0}}{\partial I}(a)-I+1,0\right)
$$

and

$$
\mathbf{n}_{2}(a)=\left(\frac{\partial K}{\partial p_{1}}(a), \frac{\partial K}{\partial p_{2}}(a), \frac{\partial K}{\partial q_{1}}(a), \frac{\partial K}{\partial q_{2}}(a), \frac{\partial K}{\partial I}(a), 0\right)=\left(q_{2},-q_{1},-p_{2}, p_{1}, 0,0\right)
$$

At the resonance, the component $M_{1}\left(1, \phi_{0}, \theta_{0}\right)$ along the normal $\mathbf{n}_{1}(a)$ is again equal to

$$
M_{1}\left(1, \phi_{0}, \theta_{0}\right)=H_{1}\left(0,0,0,0,1, \theta_{0}-\mu\right)-H_{1}\left(0,0,0,0,1, \theta_{0}+\mu\right)=2 \sin \mu \sin \theta_{0} .
$$

The component $M_{2}\left(1, \phi_{0}, \theta_{0}\right)$ along the normal $\mathbf{n}_{2}(a)$ is equal to

$$
M_{2}\left(1, \phi_{0}, \theta_{0}\right)=\int_{-\infty}^{\infty}\left\langle\mathbf{n}_{2}(a(t)), \mathbf{g}(a(t))\right\rangle d t
$$

where $a(t)$ is one of the solutions (9.3) with $I=1$, and $\mathbf{g}(a(t))$ is the $\mathcal{O}(\varepsilon)$ part of the vector field (9.1), namely

$$
\mathbf{g}(a(t))=\left(-\mu^{2} q_{1} \cos \theta, 0,0,0,-\left(I-\frac{1}{2} \mu^{2} q_{1}^{2}\right) \sin \theta,-\cos \theta\right) .
$$

Thus,

$$
\begin{aligned}
M_{2}\left(1, \phi_{0}, \theta_{0}\right) & =-\int_{-\infty}^{\infty} \mu^{2} q_{1} q_{2} \cos \theta d t \\
& =-2 \mu^{2} \cos \phi_{0} \sin \phi_{0} \cos \theta_{0} \int_{-\infty}^{\infty} \operatorname{sech}^{2}(\mu t) \cos [\mu \tanh (\mu t)] d t \\
& =-\sin 2 \phi_{0} \cos \theta_{0} \int_{-\mu}^{\mu} \cos v d v \\
& =-2 \sin \mu \sin 2 \phi_{0} \cos \theta_{0} .
\end{aligned}
$$

The Melnikov vector is therefore equal to

$$
\mathbf{M}\left(I, \phi_{0}, \theta_{0}\right)=\left(2 \sin \mu \sin \theta_{0},-2 \sin \mu \sin 2 \phi_{0} \cos \theta_{0}\right) .
$$


If $\mu$ is not an integer multiple of $\pi$, this vector vanishes for $\phi_{0}=0, \phi_{0}=\pi / 2, \phi_{0}=\pi$, $\phi_{0}=3 \pi / 2$ and $\theta_{0}=0, \theta_{0}=\pi$. The Jacobian determinant $\frac{\partial\left(M_{1}, M_{2}\right)}{\partial\left(\phi_{0}, \theta_{0}\right)}$ is equal to

$$
\frac{\partial\left(M_{1}, M_{2}\right)}{\partial\left(\phi_{0}, \theta_{0}\right)}= \pm 4 \sin ^{2} \mu
$$

at those zeros, and thus it is not equal to zero, when $\mu$ is not a multiple of $\pi$. Therefore, all these zeros of the Melnikov vector are transverse. These zeros imply the existence of eight intersection surfaces of the manifolds $W^{s}\left(\mathcal{M}_{\varepsilon}\right)$ and $W^{u}\left(\mathcal{M}_{\varepsilon}\right)$ near the resonance at $I=1$.

We denote the eight intersection surfaces by $\Sigma_{\varepsilon}(0,0), \Sigma_{\varepsilon}(0, \pi), \Sigma_{\varepsilon}\left(\frac{\pi}{2}, 0\right), \Sigma_{\varepsilon}\left(\frac{\pi}{2}, \pi\right), \Sigma_{\varepsilon}(\pi, 0)$, $\Sigma_{\varepsilon}(\pi, \pi), \Sigma_{\varepsilon}\left(\frac{3 \pi}{2}, 0\right)$, and $\Sigma_{\varepsilon}\left(\frac{3 \pi}{2}, \pi\right)$, respectively. In the limit as $\varepsilon \rightarrow 0$, the surfaces $\Sigma_{0}(0,0)$, $\Sigma_{0}\left(\frac{\pi}{2}, 0\right), \Sigma_{0}(\pi, 0)$, and $\Sigma_{0}\left(\frac{3 \pi}{2}, 0\right)$ connect the straight line $\theta=-\mu$ to the straight line $\theta=\mu$, and the surfaces $\Sigma_{0}(0, \pi), \Sigma_{0}\left(\frac{\pi}{2}, \pi\right), \Sigma_{0}(\pi, \pi)$, and $\Sigma_{0}\left(\frac{3 \pi}{2}, \pi\right)$ connect the straight line $\theta=\pi-\mu$ to the straight line $\theta=\pi+\mu \equiv-\pi+\mu$ on the $h-\theta$ cylinder. In particular, instead of a symmetric pair of heteroclinic orbits connecting two unperturbed equilibria to each other, as in the example in section 8 , there is a quartet of such heteroclinic orbits that survive under perturbation in the present example. In all other respects, the geometry of orbits homoclinic to the resonance band in this example is similar to the one in section 8 . The existence of nearby chaotic dynamics may also be deduced in the same way.

\section{Acknowledgement}

The author would like to thank R. Camassa, Z. Feng, D. Hobson, D. D. Holm, T. Kaper, M. Levi, C. C. Lim, D. McLaughlin, V. Roytburd, and S. Wiggins for stimulating conversations, to the participants of the Dynamics and Combinatorics Seminar at RPI for their attention during his four lectures on this subject in February of 1992, and to the Theoretical Division and Center for Nonlinear Studies at the Los Alamos National Laboratory for their hospitality and support during the Summer of 1992. The work on this paper was partially supported by the Department of Energy grant No. DE-FG02-93ER25154. 


\section{References}

V. I. Arnold [1964]. Instability of dynamical systems with many degrees of freedom, Sov. Math. Dokl. 5, 581-585.

V. I. Arnold (Ed.) [1988], Dynamical Systems III, in Encyclopedia of Mathematical Sciences, Vol. 3, Springer-Verlag: Berlin, Heidelberg, New York, London, Paris, Tokyo.

A. R. Bishop, R. Flesch, M. G. Forest, D. W. McLaughlin, and E. A. Overman [1990]. Correlations between chaos in a perturbed sine-Gordon equation and a truncated model system, SIAM J. Math. Anal. 21, 1511-1536.

S. N. Chow, J. Hale, and J. Mallet-Paret [1980]. An example of bifurcation to homoclinic orbits, J. Diff. Eqns. 37, 351-373.

D. David, D. D. Holm, and M. V. Tratnik [1990]. Hamiltonian chaos in nonlinear optical polarization dynamics, Phys. Reports 187, 283-367.

Z. C. Feng [1990]. Private communication.

Z. C. Feng and P. R. Sethna [1989]. Symmetry-breaking bifurcations in resonant surface waves, J. Fluid. Mech. 199, 495-518.

Z. C. Feng and P. R. Sethna [1990]. Global bifurcation and chaos in parametrically forced systems with one-one resonance, Dynamics and Stability of Systems 5, 201-225.

Z. C. Feng and S. Wiggins [1992]. On the existence of chaos in a class of two-degree-offreedom, damped, parametrically forced mechanical systems with broken $O(2)$ symmetry, ZAMP 44, 201-248.

N. Fenichel [1971]. Persistence and smoothness of invariant manifolds for flows, Ind. Univ. Math. J. 21, 193-225.

N. Fenichel [1974]. Asymptotic stability with rate conditions, Ind. Univ. Math. J. 23, 1109-1137.

N. Fenichel [1977]. Asymptotic stability with rate conditions, II, Ind. Univ. Math. J. 26, 81-93.

N. Fenichel [1979]. Geometric singular perturbation theory for ordinary differential equations, J. Diff. Eqns. 31, 53-98. 
H. Goldstein [1980]. Classical Mechanics, 2nd Ed., Addison-Wesley: Reading, Massachussetts.

B. D. Greenspan and P. J. Holmes [1984]. Repeated resonance and homoclinic bifurcation in a periodically forced family of oscillators, SIAM J. Math. Anal. 15, 69-97.

X. M. Gu and P. R. Sethna [1987]. Resonant surface waves and chaotic phenomena, J. Fluid. Mech. 183, 543-565.

J. Guckenheimer and P. J. Holmes [1983]. Nonlinear Oscillations, Dynamical Systems, and Bifurcations of Vector Fields, Springer-Verlag: New York, Heidelberg, Berlin.

G. Haller and S. Wiggins [1993]. Orbits homoclinic to resonances: the Hamiltonian case, Physica D 66, 298-346.

D. D. Holm and G. Kovačič [1992]. Homoclinic chaos in a laser-matter system, Physica D 56, 270-300.

D. D. Holm, G. Kovačič and B. Sundaram [1991]. Chaotic laser-matter interaction, Phys. Lett. A 154, 346-352.

P. J. Holmes [1986]. Chaotic motions in a weakly nonlinear model for surface waves, $J$. Fluid. Mech. 162, 365-388.

P. J. Holmes and J. E. Marsden [1982]. Horseshoes in perturbation of Hamiltonian systems with two degrees of freedom, Comm. Math. Phys. 82, 523-544.

C. K. R. T. Jones and N. Kopell [1994]. Tracking invariant manifolds with differential forms in singularly perturbed systems, J. Diff. Eqns. 108, 64-88.

G. Kovačič [1992a]. Hamiltonian dynamics of orbits homoclinic to a resonance band, Phys. Lett. A 167, 137-142.

G. Kovačič [1992b]. Dissipative dynamics of orbits homoclinic to a resonance band, Phys. Lett. A 167, 143-150.

G. Kovačič [1992c]. Singular perturbation theory for homoclinic orbits in a class of nearintegrable dissipative systems, to appear in SIAM J. Math. Anal..

G. Kovačič and S. Wiggins [1992]. Orbits homoclinic to resonances with an application to chaos in a model of the forced and damped Sine-Gordon equation, Physica D 57, 185-225. 
L. M. Lerman and Ia. L. Umanski [1984]. On the existence of separatrix loops in fourdimensional systems similar to integrable Hamiltonian systems, PMM U.S.S.R. 47, 335-340.

D. McLaughlin, E. Overman, S. Wiggins, and C. Xiong [1993]. Homoclinic behavior for a 2 mode truncation of NLS, preprint.

V. K. Melnikov [1963]. On the stability of the center for time periodic perturbations, Trans. Moscow Math. 12, 1-57.

H. Poincaré [1899]. Les Méthodes Nouvelles de la Mécanique Celeste, 3 vols., GauthierVillars: Paris.

C. Robinson [1988]. Horseshoes for autonomous Hamiltonian systems using the Melnikov integral, Ergod. Th. Dynam. Sys. 8*, 395-409.

K. Sakamoto [1990]. Invariant Manifolds In Singular Perturbation Problems For Ordinary Differential Equations, Proc. Royal Soc. Edinburgh, A-Math. 116, 45-78.

S. Wiggins [1988]. Global Bifurcations and Chaos: Analytical methods, Springer-Verlag: New York.

S. Wiggins [1990]. Introduction to Applied Nonlinear Dynamical Systems and Chaos, Springer-Verlag: New York, Berlin, Heidelberg, London, Paris, Tokyo, Hong Kong.

X. L. Yang and P. R. Sethna [1991]. Local and global bifurcations in parametrically excited vibrations of nearly square plates, Int. J. Nonlin. Mech. 26, 199-220. 